CIRJE-F-881

\title{
A Discrete/Continuous Choice Model on the Nonconvex Budget Set
}

\author{
Koji Miyawaki \\ Kwansei Gakuin University \\ Yasuhiro Omori \\ University of Tokyo \\ Akira Hibiki \\ Sophia University \\ March 2013
}

CIRJE Discussion Papers can be downloaded without charge from:

http://www.cirje.e.u-tokyo.ac.jp/research/03research02dp.html

Discussion Papers are a series of manuscripts in their draft form. They are not intended for circulation or distribution except as indicated by the author. For that reason Discussion Papers may not be reproduced or distributed without the written consent of the author. 


\title{
A discrete/continuous choice model
}

\section{on the nonconvex budget set}

\author{
Koji Miyawaki $^{\mathrm{a}^{*}} \quad$ Yasuhiro Omori $^{\mathrm{b}} \quad$ Akira Hibiki $^{\mathrm{c}}$
}

February 28, 2013

${ }^{a}$ School of Economics, Kwansei Gakuin University, Hyogo 662-8501, Japan (miyawaki.koji@kwansei.ac.jp).

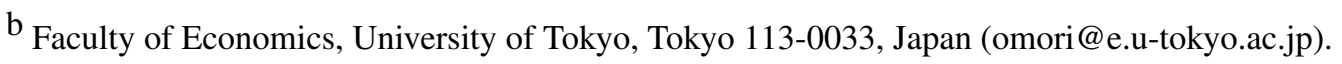

c Faculty of Economics, Sophia University, Tokyo 102-8554, Japan (hibiki@ sophia.ac.jp).

\begin{abstract}
The decreasing block rate pricing is a nonlinear price system often used for public utility services. The residential gas services in Japan and the United Kingdom are provided under this price schedule. The discrete/continuous choice approach is used to analyze the demand under decreasing block rate pricing. However, the nonlinearity problem, which has not been examined in previous studies, arises because a consumer's budget set (a set of affordable consumption amounts) is nonconvex and, hence, the resulting model includes highly nonlinear functions. To address this problem, we propose a feasible, efficient method of demand on the nonconvex budget. The advantages of our method are as follows: (i) the construction of an efficient Markov chain Monte Carlo algorithm with an efficient blanket based on the Hermite-Hadamard integral inequality and the power-mean inequality, (ii) the explicit consideration of the (highly nonlinear) separability condition, which often makes numerical likelihood maximization difficult, and
\end{abstract}

\footnotetext{
*Corresponding author: Phone:+81-798-54-6204. E-mail:miyawaki.koji@kwansei.ac.jp.
} 
(iii) the introduction of normal disturbance into the discrete/continuous choice model on the nonconvex budget set. The proposed method is applied to estimate the Japanese residential gas demand function and evaluate the effect of price schedule changes as a policy experiment.

Key words: Discrete/Continuous choice approach, Nonconvex budget set, Bayesian analysis, Residential gas demand, Hermite-Hadamard integral inequality.

JEL classification: C11, C24, D12.

\section{Introduction}

The decreasing block rate pricing is a nonlinear price system where the unit prices discontinuously decline with the quantity consumed. Figure 2(a) illustrates a typical decreasing block rate pricing. As this figure shows, there are several threshold values that divide the consumption amount and the unit prices decrease when the quantity consumed exceeds these threshold values. The residential gas services in Japan and the United Kingdom are often provided under decreasing block rate pricing (see also Section 5 for the case of Japan). Other services, such as the mobile phone service (the personal handy-phone system) in Japan and some of the residential electricity services in the United States, also employ this price system. Such a price schedule is likely to be employed partly because the production cost is decreasing in scale and partly because this system is considered to encourage a larger amount of consumption. Formal description of this price schedule is as follows.

Let $K$ be the number of blocks in the decreasing block rate pricing. In Figure 2(a), $K=3$. The consumption amount $Y$ is divided into these blocks. Let $\bar{Y}_{k}$ be the upper limit quantity for the $k$-th block $(k=0, \ldots, K)$. We set $\bar{Y}_{0} \equiv 0$ and $\bar{Y}_{K} \equiv \infty$ for simplicity. Then, each block is defined as the interval $\left[\bar{Y}_{k-1}, \bar{Y}_{k}\right)$ for $k=1, \ldots, K$. The unit prices are set in relation to these blocks and let $P_{k}$ be the unit price for the $k$-th block $(k=1, \ldots, K)$. Under decreasing block rate pricing, the unit price monotonically and discontinuously declines according to 
the blocks, that is, $P_{k+1}<P_{k}$ for $k=1, \ldots, K-1$. When, on the other hand, $P_{k}<P_{k+1}$, such a price schedule is called the increasing block rate pricing. These two price schedules are special cases of block rate pricing, where the unit prices discontinuously change with the quantity consumed. Chapter 7 of Train (1991) provides a brief microeconomic analysis of block rate pricing.

Generally, it is often the case in consumers' demand analysis to examine how consumers respond to the unit price. However, under decreasing block rate pricing, there are several unit prices depending on the consumption amount, and makes the analysis more complicated. From the microeconomics' point of view, such a response is modeled through the demand function based on the so-called discrete/continuous choice approach, which is first proposed by Burtless and Hausman (1978). ${ }^{1}$ As a consequence of this discrete/continuous choice approach, we can evaluate the social welfare, such as the compensating variation, under decreasing block rate pricing in comparison with that under uniform price system where there is only one fixed unit price. A formal presentation of this welfare measure is found in Subsection 3.2 and the empirical analysis based on the compensating variation is given in Subsection 5.3.

This demand function, however, has not been investigated in the previous empirical studies because it requires the comparison of nonlinear functions (i.e., nonlinear indirect utility functions which will be defined in Subsection 2.1 and their functional forms will be specified in Subsection 3.1). Such a nonlinearity is caused by Roy's identity which is a partial differential equation based on the consumer's behavior in the microeconomic theory. ${ }^{2}$

To avoid this nonlinearity, Blomquist and Newey (2002) proposed a nonparametric ap-

\footnotetext{
${ }^{1}$ This approach has also been used to examine a wide range of topics including housing (Lee and Trost (1978); King (1980)), transportation (Mannering and Winston (1985); Hensher and Milthorpe (1987); de Jong (1990); West (2004)), labor supply (Burtless and Hausman (1978); Burtless and Moffitt (1985)), electricity demand (Herriges and King (1994)), and water demand (Hewitt and Hanemann (1995); Olmstead, Hanemann, and Stavins (2007); Miyawaki, Omori, and Hibiki (2013)). When the budget set is convex, Miyawaki et al. (2013) proposed the appropriate estimation method. However, as we will see later, the nonconvex budget case (including the decreasing block rate pricing case) is much more difficult to estimate model parameters properly.

${ }^{2}$ We face the nonlinearity under decreasing block rate pricing, though we do not under increasing block rate pricing (see, e.g., Moffitt (1986)).
} 
proach. They analyzed the effect of tax reform in Sweden on working hours for married or cohabiting men from 20 to 60 years of age. For employees, the working time is influenced by the tax system, and it is interpreted as a block rate pricing. Then, the employee's decision about how much time to work can be considered as the problem under block rate pricing. Thus, Blomquist and Newey (2002) estimated the function of working time as a nonparametric function of the entire tax system. Though their approach is free of the nonlinearity caused by Roy's identity and of model misspecifications and distributional errors, it ignores foundational aspects of the microeconomic theory like Roy's identity. Thus, this article considers a parametric model of demand to appropriately address Roy's identity.

Previous literature (e.g., Burtless and Hausman (1978); Hausman (1980); Burtless and Moffitt (1985)) has used parametric models that are based on the discrete/continuous choice approach and applied them to estimate the effect of block rate pricing involving a two-block decreasing block rate pricing using the maximum likelihood method. ${ }^{3}$ However, two-block rate pricing is too simple for use in the analysis of real data such as Japanese residential gas data, where the number of blocks is much greater than two. (Indeed, the number of blocks is three to six depending on the gas company.) If the block structure was simplified to mimic two-block rate pricing, the estimates of the demand function as used for policymaking would be biased. Thus, we consider general multiple-block decreasing block rate pricing as a type II Tobit model subject to many nonlinear constraints (see Chapter 10 of Amemiya (1985) for the Tobit classification) and propose its Bayesian estimation method using a Markov chain Monte Carlo (MCMC) simulator with an efficient blanket.

Because the resulting statistical model includes many nonlinear constraints on model parameters (the comparison of nonlinear functions and the separability condition, which will

\footnotetext{
${ }^{3}$ Recently, Szabó (2009) proposed the maximum likelihood estimation method for general block rate pricing where the linear demand function is assumed. Szabó (2009) imposed a condition that the direct utility function is quasiconcave. This condition aims to guarantee that the underlying preference relation be strictly convex, that is, the preference relation be well-behaved. However, as stated in Hurwicz and Uzawa (1971), two more conditions (the nonnegative demand condition and the separability condition) are required for the underlying preference relation to be strictly convex. These additional conditions often make it difficult to numerically maximize the likelihood function. See Miyawaki et al. (2013) for the detailed discussion on this issue.
} 
be explained in the next paragraph), the support of the full conditional distribution for some model parameters becomes analytically intractable. One possible solution to this problem is rejection sampling. However, using a simple envelope function (or a simple blanket) for the support is extremely inefficient because the acceptance rate of the proposed samples is extremely low (see Section 4.3). Thus, this article develops an efficient blanket using two properties of convex functions: the Hermite-Hadamard integral inequality and the powermean inequality.

Our approach also has another particular advantage. The previous studies employing maximum likelihood estimation do not explicitly consider the separability condition, though this condition is necessary for the demand model under decreasing block rate pricing with more than two blocks. ${ }^{4}$ In contrast, under multiple-block decreasing block rate pricing, our statistical model includes the separability condition, which is highly nonlinear, to properly estimate the model parameters. Because of this condition, it is often difficult to numerically maximize the likelihood, and we need to pursue the Bayesian approach, using the MCMC simulator to estimate the model parameters.

Finally, we would like to note that our proposed method has an advantage over the other type of discrete/continuous choice analysis used in the context of the multinomial choice model, as in Dubin and McFadden (1984). The resulting statistical model is the same as that for demand under decreasing block rate pricing. Dubin and McFadden (1984) analyzed the joint choice of electric appliances and electricity demand using this approach and estimated the model parameters based on a combination of the maximum likelihood and the conditional expectation correction method. Their statistical model is simplified by introducing the logit error into the choice of electric appliance portfolios. However, such a specification implies the independence of irrelevant alternatives. The subsequent literature addresses this problem in two ways: by using the nested logit model (e.g., Goldberg (1998)) or by linearizing the nonlinear indirect utilities (e.g., Bernard, Bolduc, and Bélanger (1996)). Carpio,

\footnotetext{
${ }^{4}$ Miyawaki et al. (2013) dealt with this issue in the context of increasing block rate pricing. Under increasing block rate pricing, this condition is a set of linear constraints on model parameters.
} 
Wohlgenant, and Safley (2008) used a different method and applied it to the estimation of the demand for pick-your-own versus preharvested strawberries with normal error. However, their statistical model is a binary choice model: thus, they do not consider the separability condition. Therefore, this article is the first study to propose the discrete/continuous choice model on the nonconvex budget set with normal disturbance.

In presenting a parametric model for demand under decreasing block rate pricing, this article proposes the use of Bayesian analysis to make the following contributions: (i) the construction of an efficient MCMC algorithm with an efficient blanket based on the HermiteHadamard integral inequality and the power-mean inequality: (ii) the explicit consideration of the (highly nonlinear) separability condition, which often makes numerical likelihood maximization difficult: and (iii) the introduction of normal disturbance into the discrete/continuous choice model on the nonconvex budget set.

Using the proposed method, we analyze the residential gas demand function and evaluate the effect of price schedule changes. The substitution between residential gas and electricity will be left for the future work because our main interest is the demand function under decreasing block rate pricing.

This article is organized as follows. Section 2 explains the consumer's choice problem under one fixed unit price, describes that under the decreasing block rate pricing, and derives the demand function under decreasing block rate pricing based on the discrete/continuous choice approach. Then, Section 3 describes the corresponding statistical model and its likelihood function, with the discussion of the separability condition. In Section 4, we discuss the Bayesian approach and its MCMC algorithm with an efficient blanket. We also evaluate the adequacy of the proposed blankets. Section 5 estimates the Japanese residential gas demand function and evaluates the effect of price schedule changes. Section 6 concludes the study. 


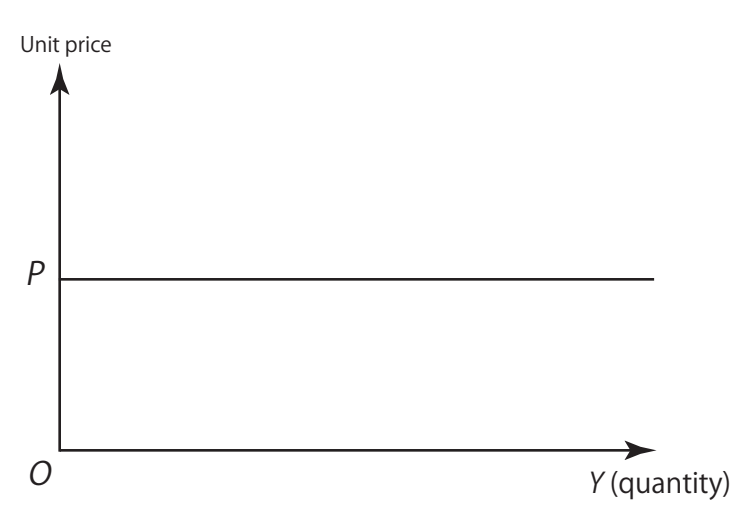

(a) Price schedule.

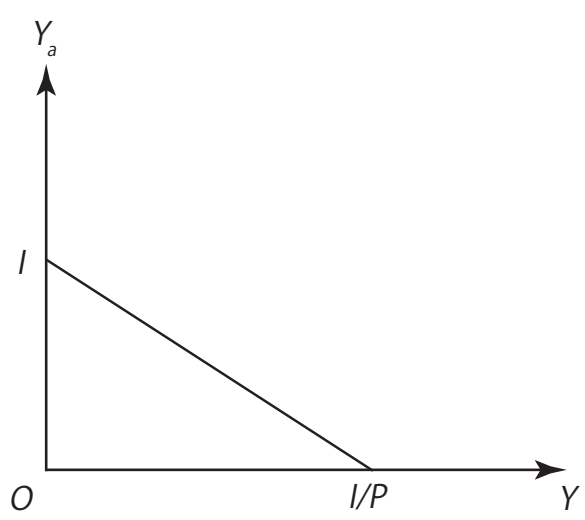

(b) Budget set.

Figure 1: A uniform price system.

\section{Demand function under decreasing block rate pricing}

\subsection{Consumer's problem under uniform price system}

This subsection describes the consumer's choice problem under uniform price system and introduces several terminology that is common in microeconomics.

Consider a consumer's choice problem between two goods, that is, the consumer need to decide the consumption amount for each good. In this subsection, both goods are supplied with one fixed unit prices. Because there are only two goods, it is sufficient to consider a (relative) unit price $P$ for one good. The unit price for the other good is normalized to one. Given the total income $I$, we define the budget set for the consumer. The budget set is a set of consumption amounts which the consumer can afford. More precisely,

$$
\left\{\left(Y, Y_{a}\right) \mid P Y+Y_{a} \leq I, Y \geq 0, Y_{a} \geq 0\right\},
$$

where $Y$ and $Y_{a}$ are the consumption amounts for goods with the unit price $P$ and one, respectively. Figure 1(a) and Figure 1(b) shows the price schedule for $Y$ and the budget set given above, respectively.

In the classical demand theory, the consumer's optimal consumption amount is deter- 
mined by maximizing its utility subject to the budget set. The utility is a measure that compares possible choices and is usually represented by a real-valued function of these choices. Let $U\left(Y, Y_{a}\right)$ be the well-defined utility function of the consumption amounts. Then, the utility maximization problem is defined as

$$
\max _{Y, Y_{a}} U\left(Y, Y_{a}\right) \quad \text { subject to } P Y+Y_{a} \leq I
$$

The solution is called the demand functions for these goods denoted by $Y(P, I)$ and $Y_{a}(P, I)$. The maximum is termed as the indirect utility function represented by $V(P, I)$. These two functions are related by the so-called Roy's identity, which is given by

$$
Y(P, I)=-\frac{\partial V(P, I) / \partial P}{\partial V(P, I) / \partial I}
$$

See, e.g., Proposition 3.4.G of Mas-Colell, Whinston, and Green (1995) for the derivation of this identity.

\subsection{Consumer's problem under decreasing block rate pricing}

This subsection examines the consumer's choice problem under decreasing block rate pricing. Suppose there are two goods: a good that is provided under decreasing block rate pricing and the numeraire good. The numeraire good represents all the other good except the good under decreasing block rate pricing and its price is normalized to one. Let $Y$ be the demand for a good under decreasing block rate pricing. Then, because the unit price declines as the consumption amount grows, the budget set becomes nonconvex (see Figure 2(b) for the three-block case).

To derive the demand function under decreasing block rate pricing, it is popular to use the so-called discrete/continuous choice approach (see, e.g., Moffitt (1986)). This approach is a two-step procedure used to solve the utility maximization problem under block rate pricing. For each $k$-th block, let $Q_{k}=I-F C-\sum_{j=1}^{k-1}\left(P_{j}-P_{j+1}\right) \bar{Y}_{j}$, where $F C$ is the minimum access 


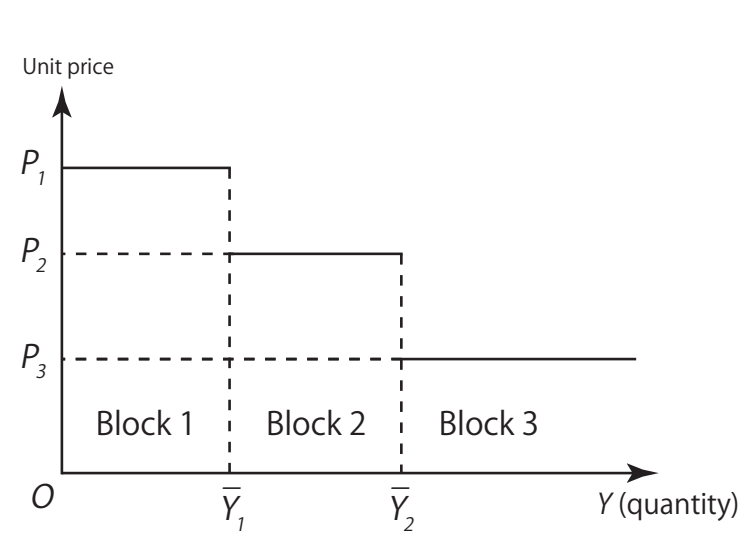

(a) Price schedule.

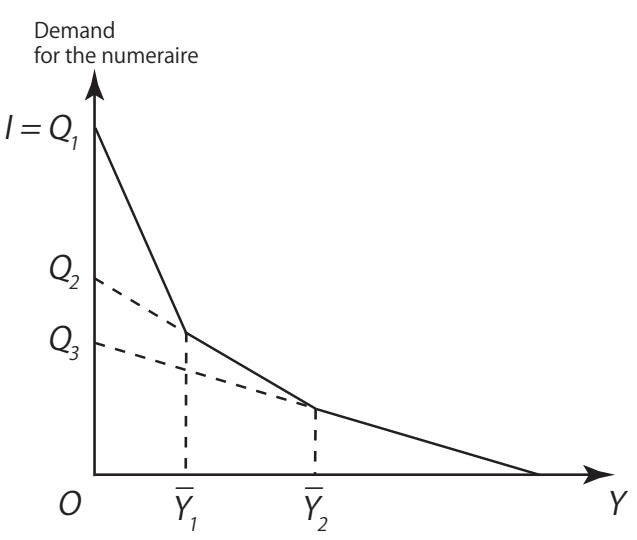

(b) Budget set.

Figure 2: A three-block decreasing block rate pricing $(K=3)$.

charge as the fixed cost. This variable is called the virtual income for the $k$-th block. We note that $Q_{k+1}<Q_{k}$ for $k=1, \ldots, K-1$ (see also Figure 2(b)).

Then, under decreasing block rate pricing, the discrete/continuous choice approach is described as follows.

Step 1. For each $k$-th block $(k=1, \ldots, K)$, maximize the utility under the uniform price system, where a consumer faces the single unit price $P_{k}$ and its corresponding virtual income $Q_{k}$. As the solution and maximum, we obtain the demand function $Y_{k}$ and the indirect utility function $V_{k}$, respectively.

Step 2. Find the block $k$ such that $V_{k}=\max _{j} V_{j}$. Then, $Y_{k}$ is the optimal demand.

In Step 1, both the price and the virtual income are given as constants. Thus, this step can be interpreted as the consumer's choice problem under uniform price system with the unit price $P_{k}$ and the virtual income $Q_{k}$, which has been described in the previous subsection. The obtained solution and maximum in this step are called the conditional demand and the conditional indirect utility, respectively, because they are derived by fixing the block choice $k$.

Finally, by following the above two steps, we obtain the demand function under decreas- 
ing block rate pricing:

$$
Y=Y_{k}, \quad V_{k}=\max _{j} V_{j}
$$

\section{Type II Tobit model with nonlinear indirect utility com- parisons}

\subsection{Log-linear demand specification}

To derive the statistical form to be used for the empirical analysis, we need to specify the functional form of the conditional demand or the conditional indirect utility (see equation (3)). Following the discussion by Hausman (1985), this article assume the conditional demand to be linear in logarithm. ${ }^{5}$ That is,

$$
\ln Y_{k}=\beta_{1} \ln P_{k}+\beta_{2} \ln Q_{k} .
$$

The log-linear function is popular in the analysis of demand under block rate pricing, because $\beta_{1}$ and $\beta_{2}$ can be directly interpreted as price and (virtual) income elasticity, respectively, conditional on block choice (see, e.g., Hewitt and Hanemann (1995); Olmstead et al. (2007)). The price elasticity, for example, is the percentage change in demand with respect to a percentage change in price. These elasticity parameters play an important role in the microeconomic theory and in the policy-making.

After specifying the conditional demand function, Roy's identity implies

$$
V_{k}=-\frac{P_{k}^{1+\beta_{1}}}{1+\beta_{1}}+\frac{Q_{k}^{1-\beta_{2}}}{1-\beta_{2}},
$$

where $\beta_{1} \neq-1$ and $\beta_{2} \neq 1$, as derived in Burtless and Hausman (1978). Plugging equations

\footnotetext{
${ }^{5}$ This functional form implicitly assumes that $P_{k}>0$ and $Q_{k}>0$ for all $k$. Under decreasing block rate pricing, these assumptions are equivalent to $P_{K}>0$ and $Q_{K}>0$.
} 
(5) and (6) into equation (4), we have the demand function under decreasing block rate pricing based on the discrete/continuous choice approach. ${ }^{6}$

We note that this theoretical framework does not exclude cases in which multiple blocks are simultaneously optimal. Such a case is excluded by introducing a continuous random disturbance into the consumer's heterogeneity in preferences. Section 3 describes its specification.

Remark 1. Hanemann (1984) proposed two other demand functions that are less popular in the literature: the linear expenditure system (LES) model and the price independent generalized log-linear (PIGLOG) model.

\subsection{Compensating variation}

Because the demand function includes the (conditional) indirect utility, we can evaluate the effect of the price schedule changes on welfare using the compensating variation. The compensating variation is a quantitative measure of welfare changes due to the price changes and is defined as the difference between the current income and the income required to attain the current utility level under the new price. The amount of positive (negative) difference can be interpreted as the degree of improvement (decline) in consumer welfare under the new price (see Chapter 3 of Mas-Colell et al. (1995) for a general discussion of the compensating variation).

For the case of decreasing block rate pricing, the compensating variation is derived as follows. Let $P=\left\{\left\{P_{k}, \bar{Y}_{k}\right\}_{k=1}^{K-1}, P_{K}, F C\right\}$ and $P^{\prime}=\left\{\left\{P_{k}^{\prime}, \bar{Y}_{k}^{\prime}\right\}_{k=1}^{K^{\prime}-1}, P_{K^{\prime}}^{\prime}, F C^{\prime}\right\}$ denote the current and the suppositional price schedule, respectively. Then, by solving

$$
V=\left(\text { the right hand side of equation (6) evaluated with } P^{\prime}\right. \text { ), }
$$

\footnotetext{
${ }^{6}$ As pointed out in Hausman (1985), our approach that involves deciding the demand function first and deriving its corresponding indirect utility function has two advantages: (i) we can flexibly choose the functional form of the demand function based on the empirical dataset, and (ii) the stochastic specification becomes convenient.
} 
for $I$, where $V$ is a certain utility level, we obtain the expenditure at the certain utility level under the suppositional price schedule $P^{\prime}$, which is given by

$$
E_{k^{\prime}}\left(P^{\prime}, V\right)=\left[\left(1-\beta_{2}\right)\left\{V+\frac{\left(P_{k^{\prime}}^{\prime}\right)^{1+\beta_{1}}}{1+\beta_{1}}\right\}\right]^{1 /\left(1-\beta_{2}\right)}+F C^{\prime}+\sum_{j=1}^{k^{\prime}-1}\left(P_{j}^{\prime}-P_{j+1}^{\prime}\right) \bar{Y}_{j}^{\prime}
$$

where $k^{\prime}=\operatorname{argmax}_{j} V_{j}^{\prime}$ and $V_{j}^{\prime}$ is the (suppositional) indirect utility conditional on the $j$-th block under $P^{\prime}$ (see Hausman (1981) for the case in which there is a single unit price). With equation (8), the compensating variation is defined as

$$
C V=I-E_{k^{\prime}}\left(P^{\prime}, V_{k}\right)
$$

where $k=\operatorname{argmax}_{j} V_{j}$ and $V_{j}$ is the $j$-th (current) conditional indirect utility under $P$.

When we assume $P^{\prime}$ to be the uniform price system, that is, $P^{\prime}=\left\{P^{*}, F C^{*}\right\}$, we have

$$
E_{k^{\prime}}\left(P^{\prime}, V\right)=\left[\left(1-\beta_{2}\right)\left\{V+\frac{\left(P^{*}\right)^{1+\beta_{1}}}{1+\beta_{1}}\right\}\right]^{1 /\left(1-\beta_{2}\right)}+F C^{*}
$$

The conditional indirect utility under $P$ is given by equation (6). Therefore, the compensating variation is calculated as

$$
C V=I-\left[\left(1-\beta_{2}\right)\left\{\frac{\left(P^{*}\right)^{1+\beta_{1}}-P_{k}^{1+\beta_{1}}}{1+\beta_{1}}+\frac{Q_{k}^{1-\beta_{2}}}{1-\beta_{2}}\right\}\right]^{1 /\left(1-\beta_{2}\right)}-F C^{*}
$$

In subsection 5.3, we will conduct the welfare analysis based on the compensating variation using the empirical data.

Remark 2. Another welfare measure is the equivalent variation, which is given by

$$
E V=E_{k}\left(P, V_{k^{\prime}}^{\prime}\right)-I
$$

Because both $E V$ and $C V$ show similar patterns with our empirical dataset, the discussion 
and the results of $E V$ are suppressed.

\subsection{Statistical model}

This subsection describes a statistical model that is a nonlinear type II Tobit model based on the theoretical framework with equations (4)-(6). There are $n$ consumers. Let subscript $i$ denote the consumer $i(i=1, \ldots, n)$ and let $\left(y_{i}, y_{i k}, p_{i k}, q_{i k}\right)=\left(\log Y_{i}, \log Y_{i k}, \log P_{i k}, \log Q_{i k}\right)$.

Then, the statistical model for the demand function under decreasing block rate pricing is given by

$$
y_{i}=y_{i s_{i}^{*}}+w_{i}^{*}+u_{i}, \quad u_{i} \sim \text { i.i.d. } N\left(0, \sigma_{u}^{2}\right),
$$

where

$$
\begin{aligned}
y_{i s_{i}^{*}} & =\beta_{1} p_{i s_{i}^{*}}+\beta_{2} q_{i s_{i}^{*}},{ }^{7} \\
w_{i}^{*} & =z_{i}^{\prime} \boldsymbol{\delta}+v_{i}, \quad v_{i} \sim \text { i.i.d. } N\left(0, \sigma_{v}^{2}\right), \\
s_{i}^{*} & =k, \quad \text { if } w_{i}^{*} \in R_{i k}=\left\{w_{i}^{*} \mid V_{i k}>V_{i j} \text { for } k \neq j\right\} \text { and } k=1, \ldots, K_{i}, \\
V_{i k} & =-\exp \left(w_{i}^{*}\right) \frac{P_{i k}^{1+\beta_{1}}}{1+\beta_{1}}+\frac{Q_{i k}^{1-\beta_{2}}}{1-\beta_{2}},
\end{aligned}
$$

$\beta_{1} \neq-1$, and $\beta_{2} \neq 1$.

In this statistical model, there are three components in addition to the theoretical framework with equations (4)-(6). The first component is $w_{i}^{*}$, which represents the consumer's heterogeneity in preferences. We introduce a hierarchical structure into the heterogeneity and assume it to be linear in the $d$-dimensional covariate vector $z_{i}$ with its corresponding coefficient vector $\boldsymbol{\delta}$. The disturbance $v_{i}$ of the heterogeneity is normally distributed with a mean of 0 and a variance of $\sigma_{v}^{2}$.

\footnotetext{
${ }^{7}$ Because of the log-linear function in (13), we require $P_{i K_{i}}>0$ and $Q_{i K_{i}}>0$ for all $i$. In our empirical dataset, there are no households whose $Q_{i K_{i}} \leq 0$.
} 
There are two following motivations for the introduction of this term. At first, as thoroughly discussed in Moffitt (1986), this term is introduced to explain unobserved tastes included in the utility function. It is natural to assume that the utility function may vary across consumers due to their unmeasured individual attributes. Then, the solutions to the utility maximization problem (i.e., the optimal demands) will differ among consumers even if they face the same price structure and earn the same income level.

Next motivation is to impose zero probability on the multiple optima to the utility maximization problem. Let us solve the comparison of conditional indirect utilities with respect to heterogeneity because the indirect utility conditional on the block choice is derived from the sum of $y_{i k}$ and $w_{i}^{*}$ using Roy's identity. The resulting interval is called the heterogeneity interval and is denoted by $R_{i k}$. The explicit formula for the heterogeneity interval is given in Appendix A.1.

To be rigorous, this interval must be $\bar{R}_{i k}=\left\{w_{i}^{*} \mid V_{i k}=\max _{j} V_{i j}\right\}$, where a tie among the conditional indirect utilities is allowed. Clearly, $R_{i k} \subseteq \bar{R}_{i k}$. However, because the set, $V_{i k}=V_{i j}$ $(j \neq k)$, has a probability of zero in our statistical model, we replace $\bar{R}_{i k}$ with $R_{i k}$. This zero probability implies that the statistical model excludes the multiple optima. The reason is as follows. Conditional on $\beta_{1}$ and $\beta_{2}$, the condition $V_{i k}=V_{i j}$ leads to the condition that $w_{i}^{*}$ must equal to a certain real value, $\ln E_{k j}$, which is derived in Appendix A.1. Because $w_{i}^{*}$ is a continuous random variable, this condition has a zero probability.

The second component is the state variable, $s_{i}^{*}$, and we can use the data augmentation method to estimate the model parameters (see Tanner and Wong (1987) for more information on this method). The $s_{i}^{*}$ is a discrete latent variable that takes one of the values from 1 to $K_{i}$ and indicates the optimal block for the $i$-th consumer.

The third component is the measurement error $u_{i}$ for demand that follows a normal distribution with a mean of 0 and a variance of $\sigma_{u}^{2}$. This term is assumed to be independent of $v_{i}$. As discussed in Hausman (1985), $u_{i}$ also represents an optimization error by the consumer and a misspecification error by the statistician. Furthermore, Moffitt (1986) pointed out that 
it is expected to give nonzero probability on the consumption amount between heterogeneity intervals (see also Figure 6 of Moffitt (1986)). When the measurement error is excluded from the model, such a consumption amount will not be observed because the upper limit of the $k$-th heterogeneity interval is less than the lower limit of the $k+1$-st interval. This situation is alleviated by the introduction of the error term.

We refer to the identification problem of two errors: $u_{i}$ for the observed demand and $v_{i}$ for heterogeneity. They cannot be fully identified unless there is additional information through the prior distribution about these errors because there is only one equation for them: $y_{i}=y_{i s_{i}^{*}}+w_{i}^{*}+u_{i}$. Although there is the identification problem, the two-error component model is preferred because of its features described above.

\subsection{Likelihood function subject to many nonlinear constraints}

The likelihood function augmented by the latent variables is given by

$$
\begin{array}{r}
f\left(y_{i}, s_{i}^{*}, w_{i}^{*} \mid \boldsymbol{\beta}, \boldsymbol{\delta}, \sigma_{u}^{2}, \sigma_{v}^{2}\right) \\
\propto\left(\sigma_{u} \sigma_{v}\right)^{-1} \exp \left[-\frac{1}{2}\left\{\sigma_{u}^{-2}\left(y_{i}-y_{i s_{i}^{*}}-w_{i}^{*}\right)^{2}+\sigma_{v}^{-2}\left(w_{i}^{*}-\boldsymbol{z}_{i}^{\prime} \boldsymbol{\delta}\right)^{2}\right\}\right] I\left(w_{i}^{*} \in R_{i s_{i}^{*}}\right) \\
\times \prod_{k=2}^{K_{i}-1} I\left(R L_{i k} \leq R U_{i k}\right),
\end{array}
$$

where $\boldsymbol{\beta} \equiv\left(\beta_{1}, \beta_{2}\right)^{\prime}$ and $I(A)$ is the indicator function: $I(A)=1$ if $A$ is true and $I(A)=0$ otherwise. $R L_{i k}$ and $R U_{i k}$ are the respective lower and upper limits of the heterogeneity interval $R_{i k}$, and their definitions are given in equation (34) in Appendix A.1. Because we take a Bayesian approach as described later and treat $\boldsymbol{\beta}$ as a continuous random vector, the conditions $\beta_{1} \neq-1$ and $\beta_{2} \neq 1$ are omitted hereafter.

The last term, the product of the $K_{i}-2$ indicator functions, is the condition that the heterogeneity intervals are separable, that is, $R_{i k} \neq \emptyset$ (for all $k$ ). We call this condition the separability condition. This condition is a set of nonlinear constraints on $\beta_{1}$ and $\beta_{2}$, and the 


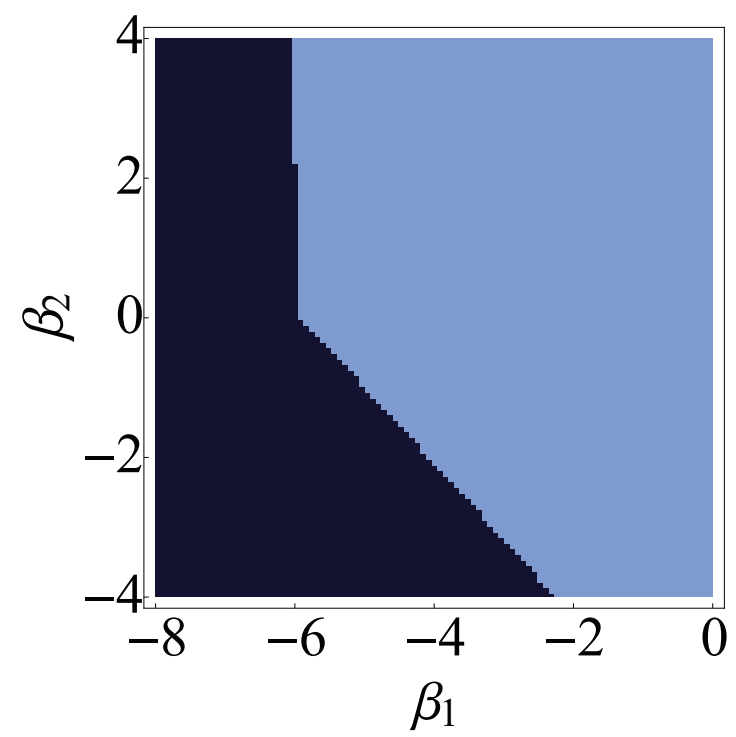

Figure 3: Region implied by the separability condition.

number of nonlinear constraints increases as the number of observations and blocks grows. Because of this condition, it is often difficult to numerically maximize the likelihood.

Figure 3 is included to show how the separability condition restricts $\left(\beta_{1}, \beta_{2}\right)$ by using the empirical dataset. Because the separability condition is analytically intractable, each point is checked whether it satisfies the condition to draw this figure. The light blue area is the area in which the separability condition holds, whereas the deep blue area is the area in which it does not. We can see that the separability condition simulated by the empirical dataset imposes nonlinear (piecewise-linear) constraints on $\left(\beta_{1}, \beta_{2}\right)$.

In general, when we analyze the multinomial choice model, such a condition is always required so that every choice is separable. Similarly, Miyawaki et al. (2013) analyzed the demand model under increasing block rate pricing, which is another multinomial choice model, and explicitly considered the requirement that the choice intervals be separable. In this case, the separability condition is a set of linear constraints on elasticity parameters. Furthermore, the separability condition is one of the sufficient conditions to make the underlying preference relation strictly convex (see Hurwicz and Uzawa (1971)). The separability condition is illustrated in the next subsection. 
We refer to the identification problem of two errors: $u_{i}$ for the observed demand and $v_{i}$ for heterogeneity. They cannot be fully identified unless there is additional information through the prior distribution about these errors because there is only one equation for them: $y_{i}=y_{i s_{i}^{*}}+w_{i}^{*}+u_{i}$.

\section{Efficient MCMC simulator based on two inequalities}

\subsection{Prior-Posterior analysis}

This article assumes the following proper prior distributions.

$$
\begin{array}{ll}
\beta_{j} \mid \sigma_{u}^{2} \sim T N_{B_{j}}\left(\mu_{\beta_{j}, 0}, \sigma_{u}^{2} \sigma_{\beta_{j}, 0}^{2}\right),(j=1,2), & \sigma_{u}^{2} \sim I G\left(\frac{n_{u, 0}}{2}, \frac{S_{u, 0}}{2}\right), \\
\boldsymbol{\delta} \mid \sigma_{v}^{2} \sim N_{d}\left(\boldsymbol{\mu}_{\boldsymbol{\delta}, 0}, \sigma_{v}^{2} \boldsymbol{\Sigma}_{\boldsymbol{\delta}, 0}\right), & \sigma_{v}^{2} \sim I G\left(\frac{n_{v, 0}}{2}, \frac{S_{v, 0}}{2}\right) .
\end{array}
$$

Conditional on $\sigma_{u}^{2}, \beta_{j}$ follows the truncated normal distribution with mean $\mu_{\beta_{j}, 0}$, variance $\sigma_{u}^{2} \sigma_{\beta_{j}, 0}^{2}$, and support $B_{j}=\left[l_{j}, m_{j}\right](j=1,2)$. Conditional on $\sigma_{v}^{2}, \boldsymbol{\delta}$ follows the $d$-dimensional multivariate normal distribution with mean vector $\boldsymbol{\mu}_{\delta, 0}$ and covariance matrix $\sigma_{v}^{2} \Sigma_{\delta, 0}$. The variance parameter $\sigma_{j}$ follows the inverse gamma distribution with parameters $n_{j, 0} / 2$ and $S_{j, 0} / 2(j=u, v)$. Its mean and variance are $S_{j, 0} /\left(n_{j, 0}-2\right)$ for $n_{j, 0}>2$ and $2 S_{j, 0}^{2} /\left\{\left(n_{j, 0}-\right.\right.$ $\left.2)^{2}\left(n_{j, 0}-4\right)\right\}$ for $n_{j, 0}>4$, respectively. The support of $\beta_{j}(j=1,2)$ reflects our prior knowledge. To elicit the prior distribution, one can make use of knowledge based on demand theory or utilize the estimates obtained from a similar population (see Subsection 5.2).

Let $\pi\left(\boldsymbol{\beta}, \boldsymbol{\delta}, \sigma_{u}^{2}, \sigma_{v}^{2}\right)$ be the prior density function of $\left(\boldsymbol{\beta}, \boldsymbol{\delta}, \sigma_{u}^{2}, \sigma_{v}^{2}\right)$. Then, it is straightforward to derive the posterior density function, which is given by

$$
\begin{aligned}
& \pi\left(\boldsymbol{\beta}, \boldsymbol{\delta}, \sigma_{u}^{2}, \sigma_{v}^{2}, \boldsymbol{s}^{*}, \boldsymbol{w}^{*} \mid \boldsymbol{y}\right) \propto \pi\left(\boldsymbol{\beta}, \boldsymbol{\delta}, \sigma_{u}^{2}, \sigma_{v}^{2}\right) \\
& \quad \times\left(\sigma_{u} \sigma_{v}\right)^{-n} \exp \left[-\frac{1}{2}\left\{\sigma_{u}^{-2}\left(\boldsymbol{y}-\boldsymbol{y}_{s^{*}}-\boldsymbol{w}^{*}\right)^{\prime}\left(\boldsymbol{y}-\boldsymbol{y}_{s^{*}}-\boldsymbol{w}^{*}\right)+\sigma_{v}^{-2}\left(\boldsymbol{w}^{*}-\boldsymbol{Z} \boldsymbol{\delta}\right)^{\prime}\left(\boldsymbol{w}^{*}-\boldsymbol{Z} \boldsymbol{\delta}\right)\right\}\right]
\end{aligned}
$$




$$
\times \prod_{i=1}^{n}\left\{I\left(w_{i}^{*} \in R_{i s_{i}^{*}}\right) \prod_{k=2}^{K_{i}-1} I\left(R L_{i k} \leq R U_{i k}\right)\right\},
$$

where $\boldsymbol{y}=\left(y_{1}, y_{2}, \ldots, y_{n}\right)^{\prime}, \boldsymbol{y}_{s^{*}}=\left(y_{1 s_{1}^{*}}, y_{2 s_{2}^{*}}, \ldots, y_{n s_{n}^{*}}\right)^{\prime}, \boldsymbol{s}^{*}=\left(s_{1}^{*}, s_{2}^{*}, \ldots, s_{n}^{*}\right)^{\prime}, \boldsymbol{w}^{*}=\left(w_{1}^{*}, w_{2}^{*}, \ldots, w_{n}^{*}\right)^{\prime}$, and $\boldsymbol{Z}=\left(z_{1}, z_{2}, \ldots, z_{n}\right)^{\prime}$.

To draw samples of model parameters from this posterior density function, we use the standard Gibbs sampler, the details of which are given in Appendix A.2 and the next subsection.

\subsection{Sampling $\beta_{1}$ with an efficient blanket}

The full conditional distribution of $\beta_{1}$ is the truncated normal distribution, $T N_{C_{1}}\left(\mu_{\beta_{1}, 1}, \sigma_{u}^{2} \sigma_{\beta_{1}, 1}^{2}\right)$, where

$$
\begin{aligned}
\sigma_{\beta_{1}, 1}^{-2} & =\sigma_{\beta_{1}, 0}^{-2}+\sum_{i=1}^{n}\left(p_{i s_{i}^{*}}\right)^{2}, \\
\mu_{\beta_{1}, 1} & =\sigma_{\beta_{1}, 1}^{2}\left[\sigma_{\beta_{1}, 0}^{-2} \mu_{\beta_{1}, 0}+\sum_{i=1}^{n} p_{i s_{i}^{*}}\left(y_{i}-\beta_{2} q_{i s_{i}^{*}}-w_{i}^{*}\right)\right], \\
C_{1} & =\left[\bigcap_{i=1}^{n} \bigcap_{j=1, j \neq s_{i}^{*}}^{K_{i}}\left\{\beta_{1} \mid V_{i k}>V_{i j}\right\}\right] \cap\left[\bigcap_{i=1}^{n} \bigcap_{k=2}^{K_{i}-1}\left\{\beta_{1} \mid R L_{i k} \leq R U_{i k}\right\}\right] \cap\left[l_{1}, m_{1}\right] .
\end{aligned}
$$

Because $C_{1}$ is difficult to evaluate analytically, we use rejection sampling. However, as revealed in the next subsection, a simple blanket, the envelope function in rejection sampling, is not efficient in the sense that the acceptance rate of the proposed candidate is extremely low. Therefore, we closely approximate $C_{1}$ by $\tilde{C}_{1}$, which is derived by using two properties of convex functions (the Hermite-Hadamard integral inequality and the power-mean inequality), thus improving our sampling efficiency.

First, without loss of generality, we assume that the support of the prior for $\beta_{1}$ is $B_{1}=$ $\left[l_{1}, 0\right]$. Then, we decompose $C_{1}$ into a set of larger sets and approximate them to obtain $\tilde{C}_{1}$. 
More precisely,

$$
C_{1} \subset \bigcap_{i=1}^{n} \bigcap_{j=1, j \neq s_{i}^{*}}^{K_{i}} C_{s_{i}^{*} j}^{1 i} \subset \bigcap_{i=1}^{n} \bigcap_{j=1, j \neq s_{i}^{*}}^{K_{i}} \tilde{C}_{s_{i}^{*} j}^{1 i} \equiv \tilde{C}_{1},
$$

where $C_{k j}^{1 i}=\left\{\beta_{1} \mid V_{i k}>V_{i j}\right\} \cap\left[l_{1}, 0\right]$. Third, we construct the interval $\tilde{C}_{k j}^{1 i}\left(\supset C_{k j}^{1 i}\right)$ using the following three steps.

Step 1. Apply the Hermite-Hadamard integral inequality. The Hermite-Hadamard integral inequality ${ }^{8}$ and $\beta_{1} \in\left[l_{1}, 0\right]$ imply

$$
\int_{P_{i j}}^{P_{i k}} x^{\beta_{1}} d x \geq \begin{cases}\left(P_{i k}-P_{i j}\right)\left(\frac{P_{i k}+P_{i j}}{2}\right)^{\beta_{1}}, & \text { if } k<j, \\ \left(P_{i k}-P_{i j}\right) \frac{P_{i k}^{\beta_{1}}+P_{i j}^{\beta_{1}}}{2}, & \text { if } k>j .\end{cases}
$$

Using this inequality, we have

$$
V_{i k}>V_{i j} \Longleftrightarrow a_{1}>\int_{P_{i j}}^{P_{i k}} x^{\beta_{1}} d x \Longrightarrow a_{1}>\text { (the right hand side of equation (24)), }
$$

where $a_{1}=\exp \left(-w_{i}^{*}\right)\left(1-\beta_{2}\right)^{-1}\left(Q_{i k}^{1-\beta_{2}}-Q_{i j}^{1-\beta_{2}}\right)$.

Step 2. Apply the power-mean inequality. The power-mean inequality and $\beta_{1} \in\left[l_{1}, 0\right]$ imply

$$
\left(\frac{P_{i k}^{l_{1}}+P_{i j}^{l_{1}}}{2}\right)^{1 / l_{1}}<\left(\frac{P_{i k}^{\beta_{1}}+P_{i j}^{\beta_{1}}}{2}\right)^{1 / \beta_{1}} \Longleftrightarrow \frac{P_{i k}^{\beta_{1}}+P_{i j}^{\beta_{1}}}{2}<\left(\frac{P_{i k}^{l_{1}}+P_{i j}^{l_{1}}}{2}\right)^{\beta_{1} / l_{1}} \cdot 9
$$

Step 3. Combine the above two-step results. By combining equations (25) and (27), and by rearranging these inequalities for $\beta_{1}$, we derive the closely approximated interval $\tilde{C}_{k j}^{1 i}=$

${ }^{8}$ Let $f:[a, b] \rightarrow \mathbb{R}$ be a convex function. Then,

$$
f\left(\frac{a+b}{2}\right) \leq \frac{1}{b-a} \int_{a}^{b} f(x) d x \leq \frac{f(a)+f(b)}{2} .
$$

See, for example, Niculescu and Persson (2003) for a proof. Niculescu and Persson (2003) also noted that the first (or last) inequality can define the convex function itself.

${ }^{9}$ See, for example, Chapter 2 of Hardy, Littlewood, and Pólya (1952) for a proof of the power-mean inequality. This equivalence also uses the fact that $f(x)=x^{\beta_{1}}\left(\beta_{1} \in\left[l_{1}, 0\right]\right)$ is decreasing as $x(>0)$ increases. 


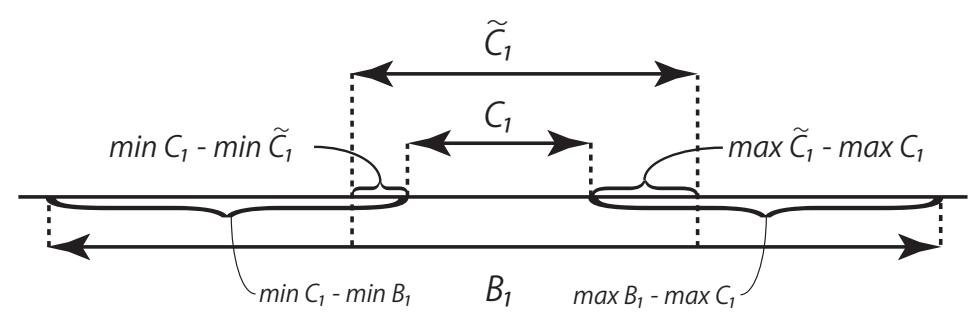

Figure 4: Relationships among $C_{1}, \tilde{C}_{1}$, and $B_{1}$.

$\tilde{C}_{k j}^{\star 1 i} \cap\left[l_{1}, 0\right]$, where

$$
\tilde{C}_{k j}^{\star 1 i}= \begin{cases}\left(-\infty, b_{1} / \bar{p}(1)\right), & \text { if } k<j \text { and } \bar{p}(1)>0, \\ (-\infty, \infty), & \text { if } k<j \text { and } \bar{p}(1)=0, \\ \left(b_{1} / \bar{p}(1), \infty\right), & \text { if } k<j \text { and } \bar{p}(1)<0, \\ \left(b_{1} / \bar{p}\left(l_{1}\right), \infty\right), & \text { if } k>j \text { and } \bar{p}\left(l_{1}\right)>0, \\ (-\infty, \infty), & \text { if } k>j \text { and } \bar{p}\left(l_{1}\right)=0, \\ \left(-\infty, b_{1} / \bar{p}\left(l_{1}\right),\right), & \text { if } k>j \text { and } \bar{p}\left(l_{1}\right)<0,\end{cases}
$$

$b_{1}=\log \left(a_{1} /\left(P_{i k}-P_{i j}\right)\right)^{10}$, and $\bar{p}(x)=x^{-1} \log \left\{\left(P_{i k}^{x}+P_{i j}^{x}\right) / 2\right\} \quad\left(x=1, l_{1}\right) . \quad$ By construction, $C_{k j}^{1 i} \subset \tilde{C}_{k j}^{1 i}$. If $P_{i K_{i}}>1$ is assumed, we have $\bar{p}(1)>\bar{p}\left(l_{1}\right)>0$, which simplifies the above expression.

Finally, by using this interval $\tilde{C}_{k j}^{1 i}$, we approximate $C_{1}$ by $\tilde{C}_{1}=\cap_{i=1}^{n} \cap_{j=1, j \neq s_{i}^{*}}^{K_{i}} \tilde{C}_{s_{i}^{*} j}^{1 i}$ as mentioned above. Figure 4 illustrates the relationships among $C_{1}, \tilde{C}_{1}$, and $B_{1}$.

With $\tilde{C}_{1}$, the sampling procedure for $\beta_{1}$ is implemented using the following two steps.

Step a. Generate $\beta_{1}^{\prime}$ from the uniform distribution on $\tilde{C}_{1}$ until it is in $C_{1}$.

\footnotetext{
${ }^{10}$ Because $a_{1} \gtrless 0$ for all $k \lessgtr j, a_{1} /\left(P_{i k}-P_{i j}\right)>0$ for any $k$ and $j(k \neq j)$.
} 
Step b. Accept $\beta_{1}^{\prime}$ with the acceptance probability $\alpha\left(\beta_{1}, \beta_{1}^{\prime}\right)$; otherwise, retain $\beta_{1}$, where

$$
\alpha\left(\beta_{1}, \beta_{1}^{\prime}\right)=\min \left[1, \frac{\phi\left\{\left(\beta_{1}^{\prime}-\mu_{\beta_{1}, 1}\right) \sigma_{u}^{-1} \sigma_{\beta_{1}, 1}^{-1}\right\}}{\phi\left\{\left(\beta_{1}-\mu_{\beta_{1}, 1}\right) \sigma_{u}^{-1} \sigma_{\beta_{1}, 1}^{-1}\right\}}\right],
$$

and $\phi(\cdot)$ is the probability density function of the standard normal distribution.

The sampling of $\beta_{2}$ is conducted in a similar manner. See Appendix A.2 for its full conditional distribution and Appendix A.3 for the derivation of its efficient blanket.

Joint sampling for $\left(\beta_{1}, \beta_{2}\right)$ is an alternative sampling algorithm. The GHK simulator (proposed by Geweke (1991), Hajivassiliou and McFadden (1998), and Keane (1994)) is a method to draw samples from the truncated multivariate normal distribution. While using this simulator could improve the sampling efficiency, the GHK simulator has disadvantages. The support of the conditional posterior distribution for $\left(\beta_{1}, \beta_{2}\right)$ is analytically difficult to evaluate because of the highly nonlinear indirect utility. Furthermore, its efficient twodimensional blanket is also difficult to construct. One of the simplest blankets is $B_{1} \times B_{2}$, which is the support of the joint prior distribution of $\left(\beta_{1}, \beta_{2}\right)$. As we see in Figure 5 and Table 1, however, this blanket is extremely inefficient with respect to the empirical dataset.

\subsection{Adequacy of the efficient blankets}

In this subsection, we evaluate the adequacy of the efficient blanket in two respects by using the Japanese residential gas demand data. The first measure is the absolute differences, $\max \tilde{C}_{j}-\max C_{j}$ and $\min C_{j}-\min \tilde{C}_{j}(j=1,2)$, and the second measure is the adequacy ratio, $\left|C_{j}\right| /\left|\tilde{C}_{j}\right|(j=1,2)$, where $|A|$ is the area of the set $A$. Figure 4 is helpful in that it clarifies what these measures mean.

Because $C_{j}$ is analytically intractable, we calculate these measures via simulation. During each step in the MCMC iterations (Appendix A.2), we obtain the approximated interval, $\tilde{C}_{j}$. Then, we compute 1,001 equispaced samples in this approximated interval and determine whether they belong to $C_{j}$. Among the samples that are in $C_{j}$, we obtain the maximum 

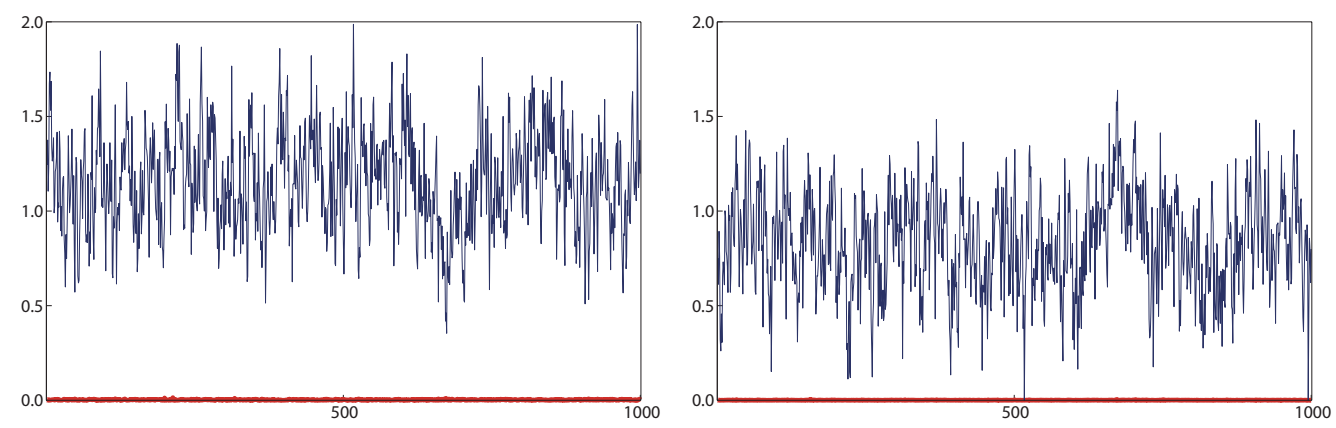

(a) $\min C_{1}-\min \tilde{C}_{1}$ (red) and $\min C_{1}-\min B_{1}$ (b) $\max \tilde{C}_{1}-\max C_{1}$ (red) and $\max B_{1}-\max C_{1}$ (blue).

(blue).
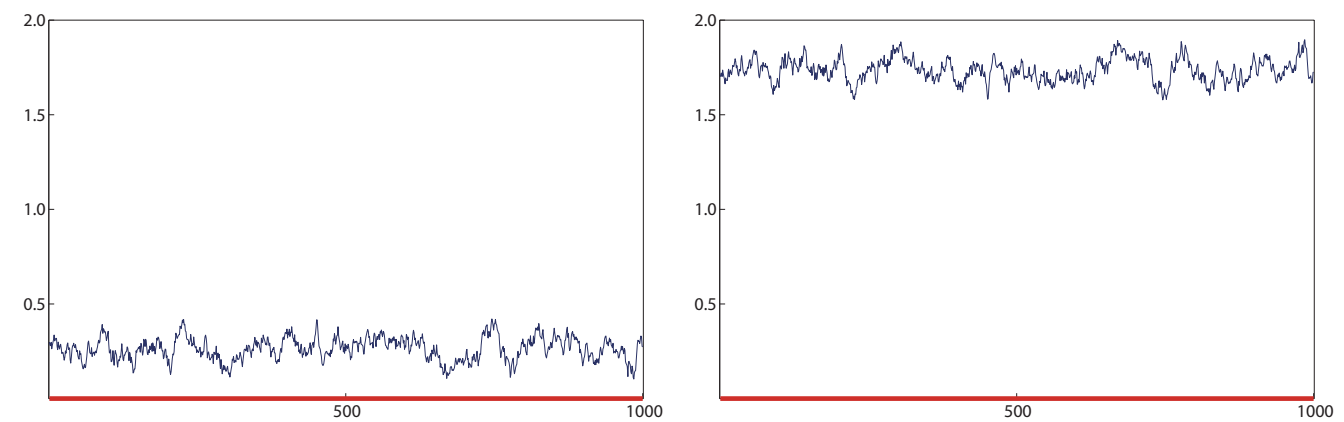

(c) $\min C_{2}-\min \tilde{C}_{2}$ (red) and $\min C_{2}-\min B_{2}$ (d) $\max \tilde{C}_{2}-\max C_{2}$ (red) and $\max B_{2}-\max C_{2}$ (blue). (blue).

Figure 5: Absolute differences.

Table 1: Adequacy ratios

\begin{tabular}{lccc}
\hline Coefficient & $\left|C_{j}\right| /\left|\tilde{C}_{j}\right|=r_{1}$ & $\left|C_{j}\right| /\left|B_{j}\right|=r_{2}$ & Efficiency ratio $\left(r_{1} / r_{2}\right)$ \\
\hline$\beta_{1}$ & $.67(.21)$ & $.0037(.0026)$ & 181 \\
$\beta_{2}$ & $1.00(.00)$ & $.0004(.0003)$ & 2,500 \\
\hline
\end{tabular}

* Standard deviations in parentheses.

and the minimum to calculate the absolute differences. Furthermore, the ratio of the number of samples that belong to $C_{j}$ to the number of those that do not is the adequacy ratio conditional on model parameters. These conditional adequacy ratios are averaged to calculate the adequacy ratio after the MCMC iterations are complete.

We calculate these two measures using the empirical dataset. The results are shown in Figure 5 and given in Table 1. Figure 5 presents time series plots of absolute differences. The red lines represent time series plots of absolute differences that calculated from our efficient blankets, whereas the blue lines are those obtained using the simple method, where $\tilde{C}_{j}$ is 
replaced by $B_{j}$. The red lines are very close to the horizontal lines at zero, which implies that the proposed efficient blankets are sufficiently close to the true sets. Table 1 indicates the adequacy ratios in the first two columns and the efficiency ratio, the ratio of two adequacy ratios, in the third column. Although the adequacy ratios of the efficient blankets differ with respect to their parameters, they are much (about 200 to 2,500 times) higher than those of the simple blanket $B_{j}$. Therefore, based on the empirical dataset, our proposed method well approximates the true regions for both $\beta_{1}$ and $\beta_{2}$.

\section{Empirical analysis and policy evaluation of residential gas demand}

\subsection{Data description}

This subsection describes the data to be used for the empirical study in the next subsection. We conducted an online survey on the Internet from June 2006 to May 2008 that was designed to analyze the water and energy consumption and the garbage emission behavior of Japanese households. The population of this survey was comprised of the households living in the Tokyo and Chiba prefectures. There were about 8.4 million households as of January 2007. Among them, 47,239 individuals were registered to the survey company, INTAGE Inc. (http://www.intage.co.jp/english/). Out of 47,239 individuals, 1,687 individuals were randomly selected. Then, out of 1,687 individuals, 1,250 participated in our survey. They were asked for household attributes such as annual income, the number of members in the household, and so on in June 2006 and April 2007. They were also asked to record their water and energy consumptions and the garbage emission behavior every month.

For the empirical study, we used the attribute data in June 2006 and the gas consumption data in January 2007. The dependent variable is the amount of gas consumption $\left(\log \mathrm{m}^{3}\right)$, which was calculated from the bill by using the corresponding gas price schedule that de- 
Table 2: Independent variables used in the gas demand function

\begin{tabular}{lcc}
\hline Coefficient & Variable & Attribute \\
\hline$\beta_{1}$ & $\left(p_{i 1}, \ldots, p_{i K_{i}}\right)$ & $\log$ of monthly unit prices of gas $\left(\log ¥ 50 / \mathrm{m}^{3}\right)$ \\
$\beta_{2}$ & $\left(q_{i 1}, \ldots, q_{i K_{i}}\right)$ & $\log$ of monthly virtual incomes $(\log ¥ 50)$ \\
\hline$\delta_{1}$ & $z_{i 1}$ & the constant \\
$\delta_{2}$ & $z_{i 2}$ & the number of members in a household (person) \\
$\delta_{3}$ & $z_{i 3}$ & the number of rooms in a home/apartment (room) \\
$\delta_{4}$ & $z_{i 4}$ & the total floor space of a home/apartment $\left(50 \mathrm{~m}^{2}\right)$ \\
\hline
\end{tabular}

Table 3: Summary statistics of the data used for the empirical study (the number of households is 473)

\begin{tabular}{lccccccc}
\hline Variable & Unit & Mean & SD & Min. & 1st quartile & 3rd quartile & Max. \\
\hline$y_{i}$ & $\log \mathrm{m}^{3}$ & 3.75 & .78 & .053 & 3.36 & 3.85 & 5.70 \\
$\log I_{i}$ & $\log ¥ 50$ & 9.22 & .56 & 7.42 & 9.03 & 9.61 & 10.82 \\
$z_{i 2}$ & person & 2.81 & 1.28 & 1 & 2 & 4 & 9 \\
$z_{i 3}$ & room & 4.09 & 1.10 & 1 & 4 & 5 & 8 \\
$z_{i 4}$ & $50 \mathrm{~m}^{2}$ & 1.54 & .74 & .20 & 1.10 & 1.80 & 8.00 \\
\hline &
\end{tabular}

pends on the area in which the individuals were living. The list of independent variables and their corresponding coefficients is given in Table 2.

The number of households is decreased from 1,250 to 473 for the reasons listed below.

- Dropped out of the survey before January 2007.

- Missing or incorrect data concerning household attributes or gas consumption.

- Use of liquefied petroleum gas. (Its price schedule is not publicly available.)

The sample selection problem will be examined later in this subsection.

For these 473 households, we conducted an empirical study that is presented in the next subsection. The first row of Table 3 gives the summary statistics of the amount of gas consumption, which is the dependent variable. All these households faced decreasing block rate pricing, and their price schedules differed depending on the cities in which they live. The price structures are shown in Figure 6, wherein the relative frequency of the number 


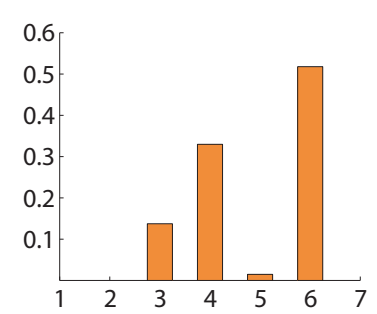

(a) Number of blocks (number).

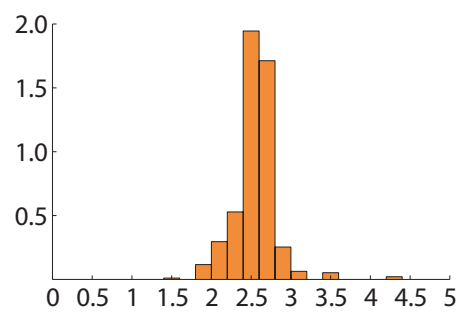

(b) Unit price $\left(¥ 50 / \mathrm{m}^{3}\right)$.

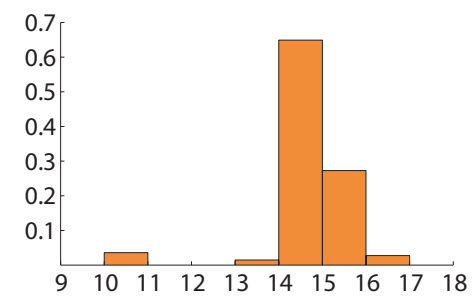

(c) Fixed gas service fee (¥50).

Figure 6: Relative frequency of the number of blocks and histograms of the unit price and the fixed gas service fee in January 2007.

of blocks, the histogram of the unit price where the gas was actually consumed, and the histogram of the fixed gas service fee are illustrated.

Because the exact annual income level is sensitive information to request, our survey divides annual income levels into eight categories: (in million yen) 0-2, 2-4, 4-6, 6-8, 8-10, 10-12, 12-15, and over 15 . Then, we ask the household its income category. The monthly income variable to be used for the empirical study is estimated using the median of the recorded income category divided by 12 . For the last category (over 15 million yen), the approximate annual income is also recorded, and we use this figure divided by 12 as the monthly income. The second row of in Table 3 summarizes the log of this income variable.

The summary statistics for the explanatory variables for heterogeneity are given in the third to the fifth rows of Table 3 . At the bottom of this table, the correlation coefficients among the explanatory variables for heterogeneity are calculated. From these values, we can establish that there is a high positive correlation between the number of rooms and the total floor space, such that either of these variables could not explain the residential gas demand.

At the end of this subsection, we discuss the sample selection bias caused by our data reduction. Table 4 gives summary statistics based on the original data. In the third column, the numbers of households to calculate these statistics are also given. For example, there are 564 households who did not drop out our survey as of January 2007 and answered the questions about gas consumption properly. These statistics are mostly similar to those given 
Table 4: Summary statistics of the original data

\begin{tabular}{lcccccc}
\hline Variable & Unit & the number of households & Mean & $S D$ & 1st quartile & 3rd quartile \\
\hline$y_{i}$ & $\log \mathrm{m}^{3}$ & 564 & 3.83 & .93 & 3.40 & 4.30 \\
$\log I_{i}$ & $\log ¥ 50$ & 1,103 & 9.17 & .56 & 9.03 & 9.62 \\
$z_{i 2}$ & person & 1,230 & 2.87 & 1.36 & 2 & 4 \\
$z_{i 3}$ & room & 1,230 & 4.13 & 1.29 & 3 & 5 \\
$z_{i 4}$ & $50 \mathrm{~m}^{2}$ & 1,230 & 1.56 & .91 & 1.00 & 1.90 \\
\hline
\end{tabular}

${ }^{*} \operatorname{Corr}\left(z_{i 1}, z_{i 2}\right)=.52, \operatorname{Corr}\left(z_{i 1}, z_{i 3}\right)=.38, \operatorname{Corr}\left(z_{i 2}, z_{i 3}\right)=.67$.

in Table 3. Therefore, it is natural to assume that the sample selection bias is small.

\subsection{Residential gas demand function}

We assume the following prior distributions.

$$
\begin{aligned}
\beta_{1} \mid \sigma_{u}^{2} \sim T N_{[-2,0]}\left(0,100 \sigma_{u}^{2}\right), & \sigma_{u}^{2} \sim I G(0.01,0.01), \\
\beta_{2} \mid \sigma_{u}^{2} \sim T N_{[0,2]}\left(0,100 \sigma_{u}^{2}\right), & \sigma_{v}^{2} \sim I G(0.01,0.01), \\
\boldsymbol{\delta} \mid \sigma_{v}^{2} \sim N_{4}\left(\mathbf{0}, 100 \sigma_{v}^{2} \boldsymbol{I}\right), &
\end{aligned}
$$

where $\boldsymbol{I}$ is the identity matrix. The truncation interval for $\beta_{j}(j=1,2)$ is elicited as follows. Because residential gas is one of the necessities for households, its demand is relatively inelastic with respect to price and income. Thus, we can expect the absolute values of $\beta_{1}$ and $\beta_{2}$ to be less than one. Furthermore, we assume negative price elasticity according to microeconomic demand theory (see, e.g., Mas-Colell et al. (1995)), and positive income elasticity according to the estimate taken from the Family Income and Expenditure Survey (FIES) conducted in 2008. The FIES survey is intended to analyze the Japanese households and estimated the expenditure elasticity for gas to be 0.29 (for households with more than two members) and away from zero at a 5\% significant level. Thus, we assume the interval $[-2,0]([0,2])$ for $\beta_{1}\left(\beta_{2}\right)$, where $-1(1)$ is included to examine whether $\beta_{1}\left(\beta_{2}\right)$ is less than -1 (more than 1). Further analysis of our empirical dataset reveals that this prior truncation area for $\boldsymbol{\beta}$ is included in the area in which the separability condition is satisfied (see Figure 
Table 5: Gas demand function

\begin{tabular}{|c|c|c|c|c|c|}
\hline Parameter & Mean & $S D$ & \multicolumn{2}{|c|}{$95 \%$ interval } & INEF \\
\hline$\beta_{1}$ (price) & -.84 & .26 & {$[-1.35$} & $-.32]$ & 136 \\
\hline$\beta_{2}$ (income) & .26 & .060 & .14 & $.38]$ & 218 \\
\hline$\delta_{1}$ & .84 & .62 & {$[-.32$} & $2.06]$ & 259 \\
\hline$\delta_{2}(1$ & .17 & .026 & .12 & $.22]$ & 11 \\
\hline & 8 & .037 & [ .11 & $.25]$ & 5 \\
\hline$\delta_{4}($ total $\mathrm{fl}$ & .038 & .052 & {$[-.067$} & $.14]$ & 6 \\
\hline & .55 & .13 & .12 & $.65]$ & 19 \\
\hline$\sigma_{v}$ (heterogeneity error) & .17 & .15 & {$[\quad .049$} & $.58]$ & 30 \\
\hline
\end{tabular}

* "SD" and "INEF" denote the posterior standard deviation and the inefficiency factor, respectively.

$3)$.

With these prior distributions, the MCMC simulation (Appendix A.2) was carried out to obtain $6 \times 10^{6}$ samples after deleting the first $6 \times 10^{5}$ samples. We reduced the obtained $6 \times 10^{6}$ samples to $10^{4}$ samples by picking up every 600 -th sample. The results are given in Table 5 and shown in Figure $7 .^{11}$

Each column of the table represents the parameter names, the posterior means, the posterior standard deviations, the $95 \%$ credible intervals, and the estimated inefficiency factors. The inefficiency factor is defined as $1+2 \sum_{j=1}^{\infty} \rho(j)$, where $\rho(j)$ is the sample autocorrelation at lag $j$, and is estimated using the spectral density. It can be interpreted as the ratio of the variance of the sample mean obtained by the MCMC draws to the variance of the sample mean by an uncorrelated Monte Carlo draw (see, e.g., Chib (2001)).

\footnotetext{
${ }^{11}$ Because our data are reduced from 1,250 to 473 , such a large data reduction would influence the obtained results. To examine the effect from this reduction, we gathered households whose dependent variables are missing but whose explanatory variables are not. The number of households then became 759. Under the same MCMC setting, we estimated the residential gas demand function. Missing dependent variables were imputed by using the model likelihood (17). Obtained results are quite similar to those given in Table 5 and shown in Figure 7, and we omit the details. Thus, the data reduction in explanatory variables does not influence the estimates of model parameters.
} 


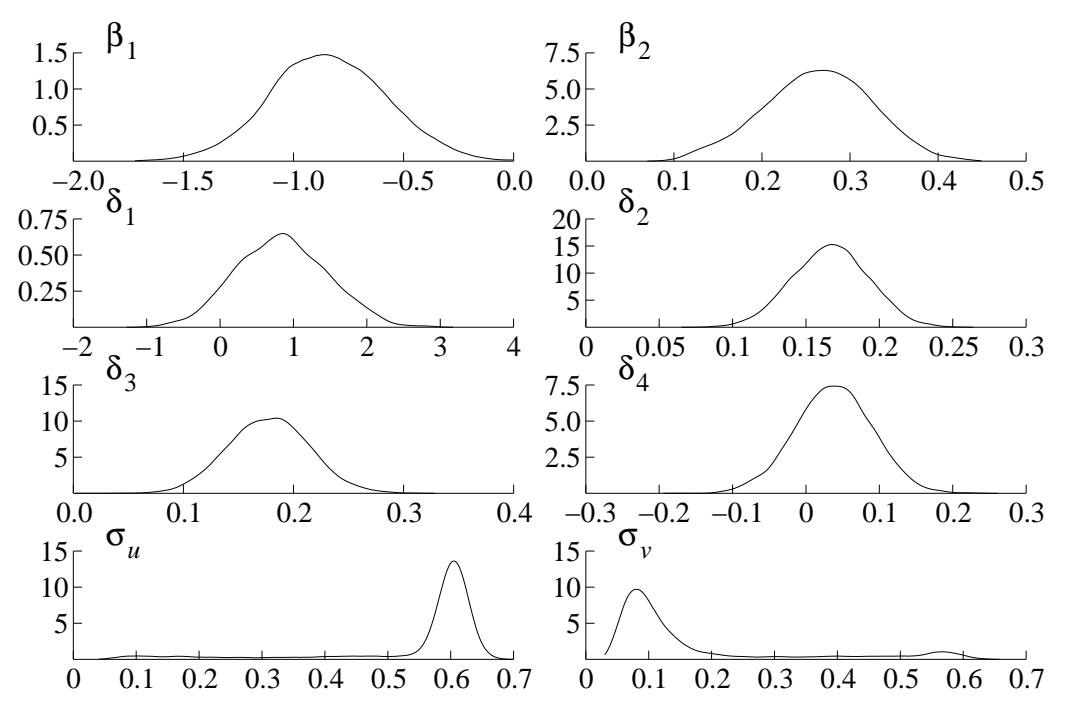

Figure 7: Marginal posterior densities.

\subsubsection{Estimates of price and income elasticities}

Price and income elasticities are highly credible to be negative and positive, respectively, in the sense that their $95 \%$ credible intervals do not include zero. Furthermore, income elasticity is highly credible to be less than one. The estimated inefficiency factors of elasticity parameters (as well as that of $\delta_{1}$ ) are much higher than other parameters. This is partly because of the tight restrictions on $\beta$ and partly because of the high correlation between $\beta_{2}$ and $\delta_{1}\left(\operatorname{Corr}\left(\beta_{2}, \delta_{1}\right)=-0.82\right)$. The other correlation coefficients are less than 0.7 in their absolute values except for that between $\sigma_{u}$ and $\sigma_{v}\left(\operatorname{Corr}\left(\sigma_{u}, \sigma_{v}\right)=-0.93\right)$.

We compared these estimates with those of previous studies. One of the classical studies of residential gas demand is the study by Balestra and Nerlove (1966). They analyzed the new gas demand using a dynamic model with random effects. Their data are the statelevel panel data for the United States during 1950-62. They estimated the (long-run) price and income elasticities to be -0.63 and 0.62 , respectively, when the depreciation rate for gas appliances is unconstrained. While the estimated income elasticity calculated by these researchers using aggregate data is larger than ours, the estimated price elasticity is similar to ours. 
Bloch (1980) also investigated residential gas demand by using the household-level data. This includes gas usage data for households living in Twin Rivers, New Jersey, during the winter months (November through April) from 1971 to 1976. The explanatory variables that Bloch (1980) used are the number of heating degree days, the price of natural gas, and the consumer price index. He found that the (long-run) price elasticity is estimated to be -0.596 or -0.224 depending on the functional form of the demand function. The former estimate is similar to our results.

\subsubsection{Other parameters}

Among the explanatory variables for heterogeneity, the number of members in a household and the number of rooms in a home are highly credible to be positive in terms of their $95 \%$ credible intervals. These factors should have a positive relationship with gas demand through water demand for the two following reasons: (1) these two variables are also credible to be positive in the Japanese residential water demand function (see Table 5 of Miyawaki et al. (2013)); and (2) in Japan, residential gas is mainly used for boiling water.

\subsection{Policy evaluation-the effect of price schedule changes}

In this subsection, we conduct a welfare analysis and evaluate the effect of price schedule changes. As the suppositional price schedules, we use the following three uniform price systems, which differ in their unit price: (unit price, fixed service fee) $=\left(¥ 50 / \mathrm{m}^{3}\right.$, ¥725), $\left(¥ 120 / \mathrm{m}^{3}, ¥ 725\right)$, and $\left(¥ 250 / \mathrm{m}^{3}, ¥ 725\right)$. These unit prices are less expensive, as high as, or more expensive than the unit price that most households are actually facing. The fixed service fee is set close to the actual fee for most households.

Figure 8 shows the effect of price changes on households in terms of compensating variation. Each boxplot is the predictive distribution of the compensating variation in one thousand yen for each household. The number of households is reduced to 90 by selecting every 5-th household. Boxplots are sorted in ascending order based on the number of members in 

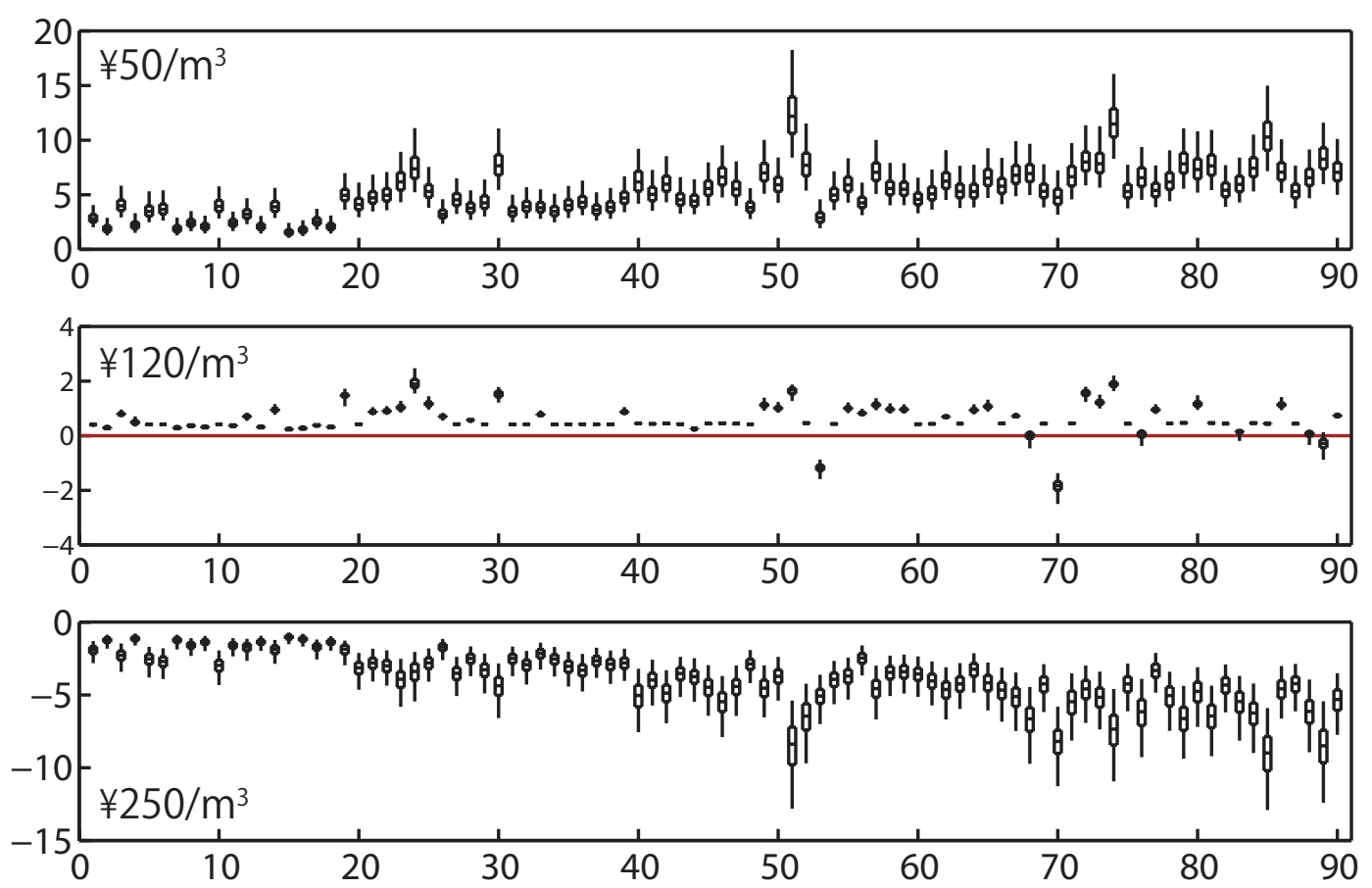

Figure 8: Boxplots of the predictive distribution of the compensating variation $\left(¥ 10^{3}\right)$. Each box represents the range between the first and third quartiles. The upper and lower whiskers denote the 95-th and 5-th percentiles, respectively.

a household.

These results are consistent with what we expect based on microeconomic theory. We observe the positive (negative) compensating variation when the unit price decreases (increases). That is, the unit price decrease (increase) implies welfare improvement (decline). However, uniform pricing itself does not seem to have a noticeable influence on compensating variation (see the panel of $¥ 120 / \mathrm{m}^{3}$ ). Furthermore, the degree of improvement (decline) is affected by explanatory variables for heterogeneity. The above panels show that the more members there are in a household, the more the compensating variation is likely to change. A similar pattern is also found with other explanatory variables for heterogeneity. 


\section{Concluding remarks}

There are many previous studies that have used the discrete/continuous choice approach in the analysis of household behaviors under block rate pricing, transportation, housing, labor supply, etc. It should be noted that the indirect utility function becomes highly nonlinear, when the budget set is nonconvex, such as in the case of decreasing block rate pricing. However, previous studies (Burtless and Hausman (1978); Hausman (1980); Burtless and Moffitt (1985)) on decreasing block rate pricing do not address this problem. Blomquist and Newey (2002) proposed a nonparametric approach to address this problem, but their approach lacks the microeconomic theoretical background. This article proposes a new Bayesian estimation method for residential gas demand on the nonconvex budget set by extending the Bayesian approach taken by Miyawaki et al. (2013), which proposed a Bayesian estimation method to analyze consumer demand under increasing block rate pricing. The advantage of our method is not only that it addresses the nonlinearity problem associated with the nonconvex budget sets but also that it incorporates the (highly nonlinear) separability condition that is necessary for the demand model under multiple-block block decreasing block rate pricing and introduces normal disturbance into the multinomial choice model.

Finally, our method has the potential to estimate the multiple residential energy expenditure function. Previous studies have focused on the cross-elasticity of electricity and gas demand (see Beierlein, Dunn, and James C. McConnon (1981); Baker, Blundell, and Micklewright (1989); Lee and Singh (1994); Maddala, Trost, Li, and Joutz (1997); Vaage (2000); Mansur, Mendelsohn, and Morrison (2008)). However, they do not take into consideration the price structure of electricity and gas services. Japanese electricity services are provided under increasing block rate pricing where the unit price increases as the volume consumed increases. Thus, by combining the proposed method and the method of Miyawaki et al. (2013) to estimate the demand function under increasing block rate pricing, we could also construct a multivariate demand function under both increasing and decreasing block rate pricing in a natural manner and estimate the residential energy demand function using the 
Bayesian approach. We will leave this for our future work.

\section{A Appendices}

\section{A.1 Heterogeneity interval}

We derive the explicit bounds of the heterogeneity interval, which is given by

$$
R_{i k}=\left\{w_{i}^{*} \mid V_{i k}>V_{i j} \text { for } j \neq k\right\}=\bigcap_{j \neq k}\left\{w_{i}^{*} \mid V_{i k}>V_{i j}\right\} .
$$

Let $D\left(x_{1}, x_{0} ; \theta\right)=\theta^{-1}\left(x_{1}^{\theta}-x_{0}^{\theta}\right)\left(x_{0}>0, x_{1}>0, \theta \neq 0\right)$. Then, $D\left(x_{1}, x_{0} ; \theta\right) \gtrless 0$ if $x_{1} \gtrless x_{0} .^{12}$

With this function, we solve $V_{i k}>V_{i j}$ for $w_{i}^{*}$.

$$
\begin{aligned}
V_{i k}>V_{i j} & \Longleftrightarrow-\exp \left(w_{i}^{*}\right) D\left(P_{i k}, P_{i j} ; 1+\beta_{1}\right)>-D\left(Q_{i k}, Q_{i j} ; 1-\beta_{2}\right) \\
& \Longleftrightarrow \begin{cases}w_{i}^{*}<\ln E_{k j}, & \text { if } k<j, \\
w_{i}^{*}>\ln E_{k j}, & \text { if } k>j,\end{cases}
\end{aligned}
$$

where $E_{k j}=D\left(Q_{i k}, Q_{i j} ; 1-\beta_{2}\right) / D\left(P_{i k}, P_{i j} ; 1+\beta_{1}\right)$. The last equivalence makes use of the property of decreasing block rate pricing: $P_{i k} \gtrless P_{i j}$ and $Q_{i k} \gtrless Q_{i j}$ if $k \lessgtr j$. Both $P_{i k}>0$ and $Q_{i k}>0$ for all $k$ because we assume the log-linear function (13). Thus, $D\left(P_{i k}, P_{i j} ; 1+\beta_{1}\right) \gtrless 0$ and $D\left(Q_{i k}, Q_{i j} ; 1-\beta_{2}\right) \gtrless 0$ if $k \lessgtr j$.

Finally, we have

$$
\begin{aligned}
R_{i 1} & =\left(-\infty, \min _{1<j} \ln E_{1 j}\right), \\
R_{i k} & =\left(\max _{k>j} \ln E_{k j}, \min _{k<j} \ln E_{k j}\right), \quad k=2, \ldots, K_{i}-1, \\
R_{i K_{i}} & =\left(\max _{K_{i}>j} \ln E_{K_{i} j}, \infty\right) .
\end{aligned}
$$

\footnotetext{
${ }^{12}$ Suppose $x_{1}>x_{0}>0$. Then, because $x_{l}^{\theta}(l=0,1)$ is decreasing (increasing) with respect to $x_{l}$ if $\theta<(>) 0$, the numerator $x_{1}^{\theta}-x_{0}^{\theta} \lessgtr 0$ if $\theta \lessgtr 0$. Therefore, $D\left(x_{1}, x_{0} ; \theta\right)>0$ if $x_{1}>x_{0}>0$. Similarly, $D\left(x_{1}, x_{0} ; \theta\right)<0$ if $x_{0}>x_{1}>0$.
} 
We note that $R_{i k} \cap R_{i j}=\emptyset(k \neq j)$.

\section{A.2 Gibbs sampler}

The Gibbs sampler is implemented in seven steps.

Step 1. Set initial values to $\left(\boldsymbol{\beta}, \boldsymbol{\delta}, \boldsymbol{s}^{*}, \boldsymbol{w}^{*}, \sigma_{u}^{2}, \sigma_{v}^{2}\right)$.

Step 2. Generate $\beta_{1}$ given $\beta_{2}, \boldsymbol{s}^{*}, \boldsymbol{w}^{*}, \sigma_{u}^{2}$.

See Subsection 4.2.

Step 3. Generate $\beta_{2}$ given $\beta_{1}, \boldsymbol{s}^{*}, \boldsymbol{w}^{*}, \sigma_{u}^{2}$.

The full conditional distribution of $\beta_{2}$ is the truncated normal distribution, $T N_{C_{2}}\left(\mu_{\beta_{2}, 2}, \sigma_{u}^{2} \sigma_{\beta_{2}, 2}^{2}\right)$, where

$$
\begin{aligned}
\sigma_{\beta_{2}, 1}^{-2} & =\sigma_{\beta_{2}, 0}^{-2}+\sum_{i=1}^{n}\left(q_{i r_{i}^{*}}\right)^{2}, \\
\mu_{\beta_{2}, 1} & =\sigma_{\beta_{2}, 1}^{2}\left[\sigma_{\beta_{2}, 0}^{-2} \mu_{\beta_{2}, 0}+\sum_{i=1}^{n} q_{i s_{i}^{*}}\left(y_{i}-\beta_{1} p_{i s_{i}^{*}}-w_{i}^{*}\right)\right], \\
C_{2} & =\left[\bigcap_{i=1}^{n} \bigcap_{j \neq s_{i}^{*}, j=1}^{K_{i}}\left\{\beta_{2} \mid V_{i k}>V_{i j}\right\}\right] \cap\left[\bigcap_{i=1}^{n} \bigcap_{k=2}^{K_{i}-1}\left\{\beta_{2} \mid R L_{i k} \leq R U_{i k}\right\}\right] \cap\left[l_{2}, m_{2}\right] .
\end{aligned}
$$

The rejection sampling with an efficient blanket is applied to obtain samples of $\beta_{2}$. The efficient blanket $\tilde{C}_{2}$ will be derived in the next appendix. The acceptance probability is given by

$$
\alpha\left(\beta_{2}, \beta_{2}^{\prime}\right)=\min \left[1, \frac{\phi\left\{\left(\beta_{2}^{\prime}-\mu_{\beta_{2}, 1}\right) \sigma_{u}^{-1} \sigma_{\beta_{2}, 1}^{-1}\right\}}{\phi\left\{\left(\beta_{2}-\mu_{\beta_{2}, 1}\right) \sigma_{u}^{-1} \sigma_{\beta_{2}, 1}^{-1}\right\}}\right] .
$$

Step 4. Generate $\left(\sigma_{v}^{2}, \delta\right)$ given $\boldsymbol{w}^{*}$.

By integrating the joint density function of $\left(\sigma_{v}^{2}, \boldsymbol{\delta}\right)$ given $\boldsymbol{w}^{*}$ over $\boldsymbol{\delta}$, we have the full conditional distribution of $\sigma_{v}^{2}$ as the inverse gamma distribution, $I G\left(n_{v, 1} / 2, S_{v, 1} / 2\right)$, where $n_{v, 1}=$ $n_{v, 0}+n$ and

$$
S_{v, 1}=S_{v, 0}+\boldsymbol{\mu}_{\delta, 0}^{\prime} \Sigma_{\delta, 0}^{-1} \boldsymbol{\mu}_{\delta, 0}+\boldsymbol{w}^{* \prime} \boldsymbol{w}^{*}-\boldsymbol{\mu}_{\boldsymbol{\delta}, 1}^{\prime} \boldsymbol{\Sigma}_{\boldsymbol{\delta}, 1}^{-1} \boldsymbol{\mu}_{\boldsymbol{\delta}, 1}
$$


Then, given $\sigma_{v}^{2}$, the full conditional distribution of $\boldsymbol{\delta}$ is the multivariate normal distribution, $N_{d}\left(\boldsymbol{\mu}_{\boldsymbol{\delta}, 1}, \sigma_{v}^{2} \boldsymbol{\Sigma}_{\boldsymbol{\delta}, 1}\right)$, where

$$
\boldsymbol{\mu}_{\boldsymbol{\delta}, 1}=\boldsymbol{\Sigma}_{\boldsymbol{\delta}, 1}\left(\boldsymbol{\Sigma}_{\boldsymbol{\delta}, 0}^{-1} \boldsymbol{\mu}_{\boldsymbol{\delta}, 0}+\boldsymbol{Z}^{\prime} \boldsymbol{w}^{*}\right), \quad \boldsymbol{\Sigma}_{\boldsymbol{\delta}, 1}^{-1}=\boldsymbol{\Sigma}_{\boldsymbol{\delta}, 0}^{-1}+\mathbf{Z}^{\prime} \boldsymbol{Z}
$$

Step 5. Generate $\left\{s_{i}^{*}, w_{i}^{*}\right\}_{i=1}^{n}$ given $\boldsymbol{\beta}, \boldsymbol{\delta}, \sigma_{u}^{2}, \sigma_{v}^{2}$.

The blocking technique is applied to draw samples of $\left(s_{i}^{*}, w_{i}^{*}\right)$. The full conditional distribution of $s_{i}^{*}$ is the multinomial distribution, the probability mass function of which is given by

$$
\pi\left(s_{i}^{*}=s \mid \boldsymbol{\beta}, \boldsymbol{\delta}, \sigma_{u}^{2}, \sigma_{v}^{2}\right) \propto\left[\Phi\left\{\tau^{-1}\left(R U_{i s}-\theta_{i s}\right)\right\}-\Phi\left\{\tau^{-1}\left(R L_{i s}-\theta_{i s}\right)\right\}\right] \exp \left(-\frac{m_{i s}}{2}\right),
$$

for $s=1, \ldots, K_{i}$, where $\tau^{2}=\left(\sigma_{u}^{-2}+\sigma_{v}^{-2}\right)^{-1}$ and

$$
\left(m_{i s}, \theta_{i s}\right)=\left(\frac{\left(\sigma_{u} \sigma_{v}\right)^{-2}\left(y_{i}-y_{i s}-z_{i}^{\prime} \boldsymbol{\delta}\right)^{2}}{\sigma_{u}^{-2}+\sigma_{v}^{-2}}, \frac{\sigma_{u}^{-2}\left(y_{i}-y_{i s}\right)+\sigma_{v}^{-2} z_{i}^{\prime} \boldsymbol{\delta}}{\sigma_{u}^{-2}+\sigma_{v}^{-2}}\right)
$$

Given $s_{i}^{*}=s$, the full conditional distribution of $w_{i}^{*}$ is the truncated normal distribution, $T N_{R_{i s}}\left(\theta_{i s}, \tau^{2}\right)$.

Step 6. Generate $\sigma_{u}^{2}$ given $\boldsymbol{\beta}, \boldsymbol{s}^{*}, \boldsymbol{w}^{*}$.

The full conditional distribution of $\sigma_{u}^{2}$ is the inverse gamma distribution, $I G\left(n_{u, 1} / 2, S_{u, 1} / 2\right)$, where $n_{u, 1}=n_{u, 0}+n+2$ and

$$
S_{u, 1}=S_{u, 0}+\left(\boldsymbol{\beta}-\boldsymbol{\mu}_{\boldsymbol{\beta}, 0}\right)^{\prime} \boldsymbol{\Sigma}_{\boldsymbol{\beta}, 0}^{-1}\left(\boldsymbol{\beta}-\boldsymbol{\mu}_{\boldsymbol{\beta}, 0}\right)+\left(\boldsymbol{y}-\boldsymbol{y}^{*}\right)^{\prime}\left(\boldsymbol{y}-\boldsymbol{y}^{*}\right) .
$$

\section{Step 7. Go to Step 2.}




\section{A.3 Efficient blanket of $C_{2}$}

We assume that the support of the prior distribution for $\beta_{2}$ is $B_{2}=\left[0, m_{2}\right]$. Let

$$
C_{k j}^{2 i}=\left\{\beta_{2} \mid V_{i k}>V_{i j}\right\} \cap\left[0, m_{2}\right] \text { and } a_{2}=\exp \left(w_{i}^{*}\right)\left(1+\beta_{1}\right)^{-1}\left(P_{i k}^{1+\beta_{1}}-P_{i j}^{1+\beta_{1}}\right) .
$$

Then, the Hermite-Hadamard integral inequality and $\beta_{2} \in\left[0, m_{2}\right]$ derive

$$
a_{2}< \begin{cases}\left(Q_{i k}-Q_{i j}\right) \frac{Q_{i k}^{-\beta_{2}}+Q_{i j}^{-\beta_{2}}}{2}, & \text { if } k<j, \\ \left(Q_{i k}-Q_{i j}\right)\left(\frac{Q_{i k}+Q_{i j}}{2}\right)^{-\beta_{2}}, & \text { if } k>j .\end{cases}
$$

By applying the power-mean inequality, we have $\tilde{C}_{k j}^{2 i}=\tilde{C}_{k j}^{\star 2 i} \cap\left[0, m_{2}\right]\left(\supset C_{k j}^{2 i}\right)$, where

$$
\tilde{C}_{k j}^{\star 2 i}= \begin{cases}\left(-\infty,-b_{2} / \bar{q}\left(-m_{2}\right)\right), & \text { if } k<j \text { and } \bar{q}\left(-m_{2}\right)>0, \\ (-\infty, \infty), & \text { if } k<j \text { and } \bar{q}\left(-m_{2}\right)=0, \\ \left(-b_{2} / \bar{q}\left(-m_{2}\right), \infty\right), & \text { if } k<j \text { and } \bar{q}\left(-m_{2}\right)<0, \\ (-\infty, \infty), & \text { if } k>j \text { and } \bar{q}(1)>0, \\ \left(-\infty,-b_{2} / \bar{q}(1)\right), & \text { if } k>j \text { and } \bar{q}(1)=0, j \text { and } \bar{q}(1)<0,\end{cases}
$$

$b_{2}=\log \left(a_{2} /\left(Q_{i k}-Q_{i j}\right)\right)$, and $\bar{q}(x)=x^{-1} \log \left\{\left(Q_{i k}^{x}+Q_{i j}^{x}\right) / 2\right\}\left(x=1,-m_{2}\right)$. If $Q_{i K_{i}}>1$ is assumed, we have $\bar{q}(1)>\bar{q}\left(-m_{2}\right)>0$, which simplifies the above expression. With this closely approximated interval $\tilde{C}_{k j}^{2 i}$, we have $\tilde{C}_{2}=\cap_{i=1}^{n} \cap_{j=1, j \neq s_{i}^{*}}^{K_{i}} \tilde{C}_{s_{i}^{*}}^{2 i}$, which includes $C_{2}$.

\section{Acknowledgement}

This work is supported by the Grants-in-Aid for Scientific Research 18330039 and by the Grant-in-Aid for Young Scientists (Start-up) 21830115 from the Japanese Ministry of Educa- 
tion, Science, Sports, Culture and Technology. The authors thank K. Fukumoto (Gakushuin University), M. Fukushige (Osaka University), K. Kakamu (Chiba University), K. Oya (Osaka University), A. C. Harvey (University of Cambridge), M. Kitahara (Tokyo Metropolitan University), N. Terui (Tohoku University), and H. K. van Dijk (Erasmus Universiteit Rotterdam) for their helpful comments. The computational results were calculated by using Ox for Linux (see Doornik (2002)).

\section{References}

Amemiya, T. (1985). Advanced Econometrics. Cambridge, Massachusetts: Harvard University Press.

Baker, P., R. Blundell, and J. Micklewright (1989). Modelling household energy expenditures using micro data. The Economic Journal 99, 720-738.

Balestra, P. and M. Nerlove (1966). Cross section and time series data in the estimation of a dynamic model: The demand for natural gas. Econometrica 34(3), 585-612.

Beierlein, J. G., J. W. Dunn, and J. James C. McConnon (1981). The demand for electricity and natural gas in the northeastern united states. Review of Economics and Statistics 63(3), 403-408.

Bernard, J.-T., D. Bolduc, and D. Bélanger (1996). Quebec residential electricity demand: a microeconometric approach. The Canadian Journal of Economics 29(1), 92-113.

Bloch, F. E. (1980). Residential demand for natural gas. Journal of Urban Economics 7, $371-383$.

Blomquist, S. and W. Newey (2002). Nonparametric estimation with nonlinear budget sets. Econometrica 70(6), 2455-2480. 
Burtless, G. and J. A. Hausman (1978). The effect of taxation on labor supply: Evaluating the Gary negative income tax experiment. Journal of Political Economy 86(6), 1103-1130.

Burtless, G. and R. A. Moffitt (1985). The joint choice of retirement age and postretirement hours of work. Journal of Labor Economics 3(2), 207-236.

Carpio, C. E., M. K. Wohlgenant, and C. D. Safley (2008). A structural econometric model of joint consumption of goods and recreational time: An application to pick-your-own fruit. American Journal of Agricultural Economics 90(3), 644-657.

Chib, S. (2001). Markov chain Monte Carlo methods: Computation and inference. In J. J. Heckman and E. Leamer (Eds.), Handbook of Econometrics, Volume 5, Chapter 57, pp. 3569-3649. Amsterdam: North-Holland.

de Jong, G. C. (1990). An indirect utility model of car ownership and private car use. European Economic Review (34), 971-985.

Doornik, J. A. (2002). Object-Oriented Matrix Programming Using Ox (3rd ed.). London: Timberlake Consultants Press and Oxford.

Dubin, J. A. and D. L. McFadden (1984). An econometric analysis of residential electric appliance holdings and consumption. Econometrica 52(2), 345-362.

Geweke, J. (1991). Efficient simulation from the multivariate normal and student-t distributions subject to linear constraints and the evaluation of constraint probabilities. In E. M. Keramidas (Ed.), Computing Science and Statistics: Proceedings of the 23rd Symposium on the Interface, pp. 571-578. Fairfax, VA: Interface Foundation of North America.

Goldberg, P. K. (1998). The effects of the corporate average fuel efficiency standards in the us. The Journal of Industrial Economics 46(1), 1-33.

Hajivassiliou, V. A. and D. L. McFadden (1998). The method of simulated scores for the estimation of ldv models. Econometrica 66(4), 863-896. 
Hanemann, W. M. (1984). Discrete/Continuous models of consumer demand. Econometrica 52(3), 541-562.

Hardy, G. H., J. E. Littlewood, and G. Pólya (1952). Inequalities (2nd ed.). Cambridge: Cambridge University Press.

Hausman, J. A. (1980). The effect of wages, taxes, and fixed costs on women's labor force participation. Journal of Public Economics 14(2), 161-194.

Hausman, J. A. (1981). Exact consumer's surplus and deadweight loss. American Economic Review 71(4), 662-676.

Hausman, J. A. (1985). The econometrics of nonlinear budget sets. Econometrica 53(6), $1255-1282$.

Hensher, D. A. and F. W. Milthorpe (1987). Selectivity correction in discrete-continuous choice analysis: With empirical evidence for vehicle choice and use. Regional Science and Urban Economics 17(1), 123-150.

Herriges, J. A. and K. K. King (1994). Residential demand for electricity under inverted block rates: Evidence from a controlled experiment. Journal of Business E Economic Statistics 12(4), 419-430.

Hewitt, J. A. and W. M. Hanemann (1995). A discrete/continuous choice approach to residential water demand under block rate pricing. Land Economics 71(2), 173-192.

Hurwicz, L. and H. Uzawa (1971). On the integrability of demand functions. In J. S. Chipman, L. Hurwicz, M. K. Richter, and H. F. Sonnenschein (Eds.), Preferences, Utility, and Demand, The Harbrace series in business and economics, Chapter 6, pp. 114-148. New York: Harcourt, Brace, Jovanovich.

Keane, M. P. (1994). A computationally practical simulation estimator for panel data. Econometrica 62(1), 95-116. 
King, M. A. (1980). An econometric model of tenure choice and demand for housing as a joint decision. Journal of Public Economics 14(2), 137-159.

Lee, L.-F. and R. P. Trost (1978). Estimation of some limited dependent variable models with application to housing demand. Journal of Econometrics 8(3), 357-382.

Lee, R.-S. and N. Singh (1994). Patterns in residential gas and electricity consumption: An econometric analysis. Journal of Business E Economic Statistics 12(2), 233-241.

Maddala, G. S., R. P. Trost, H. Li, and F. Joutz (1997). Estimation of short-run and longrun elasticities of energy demand from panel data using shrinkage estimators. Journal of Business E Economic Statistics 15(1), 90-100.

Mannering, F. and C. Winston (1985). A dynamic empirical analysis of household vehicle ownership and utilization. RAND Journal of Economics 16(2), 215-236.

Mansur, E. T., R. Mendelsohn, and W. Morrison (2008). Climate change adaptation: A study of fuel choice and consumption in the US energy sector. Journal of Environmental Economics and Management 55(2), 175-193.

Mas-Colell, A., M. D. Whinston, and J. R. Green (1995). Microecnomic Theory. New York: Oxford University Press.

Miyawaki, K., Y. Omori, and A. Hibiki (2013). Exact estimation of demand functions under block-rate pricing. Forthcoming at Econometric Reviews.

Moffitt, R. (1986). The econometrics of piecewise-linear budget constraint. Journal of Business E Economic Statistics 4(3), 317-328.

Niculescu, C. P. and L.-E. Persson (2003). Old and new on the Hermite-Hadamard inequality. Real Analysis Exchange 29(2), 663-685. 
Olmstead, S. M., W. M. Hanemann, and R. N. Stavins (2007). Water demand under alternative price structures. Journal of Environmental Economics and Management 54(2), $181-198$.

Szabó, A. (2009). The value of free water: Analyzing south africa's free basic water policy. mimeo.

Tanner, M. A. and W. H. Wong (1987). The calculation of posterior distributions by data augmentation. Journal of American Statistical Association 82(398), 528-540.

Train, K. E. (1991). Optimal Regulation: the Economic Theory of Natural Monopoly. Cambridge, Mass.: MIT Press.

Vaage, K. (2000). Heating technology and energy use: a discrete/continuous choice approach to Norwegian household energy demand. Energy Economics 22(6), 649-666.

West, S. E. (2004). Distributional effects of alternative vehicle pollution control policies. Journal of Public Economics 88(3-4), 735-757. 